\title{
Error Exponents of Typical Random Codes for the Colored Gaussian Channel *
}

\author{
Neri Merhav \\ The Andrew \& Erna Viterbi Faculty of Electrical Engineering \\ Technion - Israel Institute of Technology \\ Technion City, Haifa 32000, ISRAEL \\ E-mail: merhav@ee.technion.ac.il
}

\begin{abstract}
The error exponent of the typical random code is defined as the asymptotic normalized expectation of the logarithm of the probability of error, as opposed to the traditional definition of the random coding exponent as the normalized logarithm of the expectation of the probability of error with respect to a given ensemble of codes. For a certain ensemble of independent codewords, with a given power spectrum, and a generalized stochastic mismatched decoder, we characterize the error exponent the typical random codes (TRC) for the colored Gaussian channel, with emphasis on the range of low rates, where the TRC error exponent differs in value from the ordinary random coding error exponent. The error exponent formula, which is exponentially tight at some range of low rates, is presented as the maximum of a certain function with respect to one parameter only (in the spirit of Gallager's formulas) in the case of matched decoding, and two parameters in the case of mismatched decoding. Several aspects of the main results are discussed. These include: general properties, a parametric representation, critical rates, phase transitions, optimal input spectrum (water pouring), and comparison to the random coding exponent.
\end{abstract}

Index Terms: error exponent, typical random code, reliability function, Gaussian channel, water pouring.

${ }^{*}$ This research was supported by Israel Science Foundation (ISF) grant no. 137/18. 


\section{Introduction}

Inspired by the brief article of Barg and Forney [1], in a recent work [12], the error exponent of the typical random code (TRC) for a general discrete memoryless channel (DMC) was studied. The error exponent of the TRC was defined as the asymptotic normalized expectation of the logarithm of the probability of error, as opposed to the traditional definition of the random coding exponent as the normalized logarithm of the expectation of the probability of error with respect to the same ensemble of codes. The study of error exponents for TRCs was motivated in [12, Introduction] in several ways: (i) due to Jensen's inequality, it is never worse than the random coding error exponent. (ii) In relation to (i), it is a less pessimistic performance metric compared to the ordinary random coding exponent, especially at low rates, as it does not suffer from the problem that poor codes dominate the average error probability. (iii) Given that a certain concentration property holds, it is more relevant as a performance metric, as the code is normally assumed to be randomly selected just once, and then it is used repeatedly. (iv) It captures correctly the behavior of random-like codes [2], which are well known to be very good codes.

In [12], an exact formula for the error exponent function of the TRC was derived for a general discrete memoryless channel (DMC) under the ensemble of fixed composition codes and a class of generalized likelihood decoders (GLD's) [13], [19], [25], namely, stochastic decoders that randomly select the decoded message according to a posterior distribution (given the channel output) with a certain structure. The class of GLD's considered in [12] covers many decoders of theoretical and practical interest as special cases, including deterministic metric decoders, like the maximum likelihood (ML) decoder, the maximum mutual information (MMI) decoder, $\alpha$-decoders [4] and mismatched decoders.

While the analysis in [12] is heavily based on the method of types [3], and hence applicable, in principle, to the finite-alphabet case only, here we consider the continuous-alphabet case, and more precisely, the Gaussian case. In particular, we derive a formula for the error exponent of the TRC for the additive Gaussian channel. We begin from the additive white Gaussian noise (AWGN) channel, and then extend the scope to the colored Gaussian channel with a given channel input spectrum and noise spectrum. While we present the results for the discrete-time case, a minor modification of the error exponent formula allows to pass to the continuous-time case as well. 
It is perhaps surprising that although the finite-alphabet formula of the TRC exponent [12] is not trivial to work with (as it involves a rather computationally heavy optimization of certain functionals of probability distributions), in the Gaussian noise case considered here (and even when the noise is colored), the situation is considerably better in that sense. In particular, the resulting TRC error exponent, which is provably exact at least at some range of low rates, ${ }^{1}$ involves optimization over one parameter only in the case of a GLD with the matched (ML) decoding metric, and two parameters for a general (mismatched) GLD. Finally, we present and discuss a few byproducts of our main result. These include: a parametric representation, the zero-rate TRC exponent, the range of guaranteed tightness of our bound, the rate at which the TRC exponent meets the random coding exponent, and the optimal channel input spectrum (water-pouring).

A few words on the history of related work are in order.

In the context of bounds on the reliability function of the Gaussian channel, the first random coding bound for the discrete-time case, as well as a sphere-packing lower bound, are due to Shannon [20]. His work was followed by numerical evaluations due to Slepian [23]. Ebert [5] has derived (random-coding and expurgated) upper bounds and (sphere-packing and straight-line) lower bounds on the error probability of the parallel additive Gaussian channel, which in the long block limit, are applicable to the colored Gaussian channel by using the eigenvalue distribution theorem [8] (see also [7]). The lower bounds are based on the work of Shannon, Gallager and Berlekamp [21], which appeared a year later. Ebert's results appeared also in Gallager's book [6, Chapters 7, 8]. Viterbi [24] studied decoders with generalized decision regions for the Gaussian channel as well as other very noisy channels. During the years that have passed ever since these early works were published, a vast amount of work on improved bounds has been carried out (see, e.g., [18] for a very good survey), but most of it is of lesser direct relevance to the topic of this paper.

In the context of error exponents of TRC's, on the other hand, much less work has been done before. Prior to [12], as mentioned earlier, Barg and Forney [1] have derived, among other things, the error exponent of the TRC for the binary symmetric channel (BSC) under i.i.d. binary symmetric random coding. Nazari [16] and Nazari, Anastasopoulos and Pradhan [17] derived upper and lower

\footnotetext{
${ }^{1}$ In fact, the TRC exponent function is interesting to study primarily at low rates, as beyond a certain rate, it coincides with the ordinary random coding error exponent anyway.
} 
bounds on the TRC exponent for a general DMC under the $\alpha$-decoder of [4]. Beyond that, there has been some work in the statistical-physics literature, where TRC error exponents were analyzed for special classes of codes (like LDPC and Turbo codes) using the replica method and the cavity method - see, e.g., [9], [15], [22].

The outline of the remaining part of this paper is as follows. In Section II, we establish notation conventions (Subsection II.A), present the setup (Subsection II.B), and provide some background (Subsection II.C). In Section III, we provide the main results concerning the TRC exponent and discuss them, first for the case of the AWGN channel (Subsection III.A), and then for parallel channels and the colored Gaussian channel (Subsection III.B). In Section IV, we address the question of the optimal input spectrum for the TRC exponent. Finally, in Section V, we outline the proofs of the main results.

\section{Notation Conventions, Setup and Background}

\section{A. Notation Conventions}

Throughout the paper, random variables will be denoted by capital letters and specific values they may take will be denoted by the corresponding lower case letters. Random vectors and their realizations will be denoted, respectively, by capital letters and the corresponding lower case letters, both in the bold face font. For example, the random vector $\boldsymbol{X}=\left(X_{0}, X_{1}, \ldots, X_{n-1}\right),(n$ - positive integer) may take a specific vector value $\boldsymbol{x}=\left(x_{0}, x_{1}, \ldots, x_{n-1}\right)$ in $\mathbb{R}^{n}$. When used in the linearalgebraic context, these vectors should be thought of as column vectors, and so, when they appear with superscript $T$, they will be transformed into row vectors by transposition. Thus, $\boldsymbol{x}^{T} \boldsymbol{y}$ is understood as the inner product of $\boldsymbol{x}$ and $\boldsymbol{y}$. Probability density functions (PDFs) of sources and channels will be denoted by the letters $P$ and $Q$. The probability of an event $\mathcal{E}$ will be denoted by $\operatorname{Pr}\{\mathcal{E}\}$, and the expectation operator will be denoted by $\boldsymbol{E}\{\cdot\}$.

For two positive sequences $a_{n}$ and $b_{n}$, the notation $a_{n} \doteq b_{n}$ will stand for equality in the expo-

nential scale, that is, $\lim _{n \rightarrow \infty} \frac{1}{n} \log \frac{a_{n}}{b_{n}}=0$. Similarly, $a_{n} \leq b_{n}$ means that $\limsup _{n \rightarrow \infty} \frac{1}{n} \log \frac{a_{n}}{b_{n}} \leq 0$, and so on. Accordingly, $a_{n} \doteq e^{-n \infty}$ will mean that $a_{n}$ vanishes in a super-exponential rate. The indicator function of an event $\mathcal{E}$ will be denoted by $\mathcal{I}\{E\}$. The notation $[x]_{+}$will stand for $\max \{0, x\}$. The cardinality of a finite set $\mathcal{A}$ will be denoted by $|\mathcal{A}|$. 


\section{B. Setup}

We first describe the communication system model in the simple case of the AWGN channel, and then extend the scope to parallel Gaussian channels with application to the colored Gaussian channel.

\section{The AWGN Channel}

Consider the discrete-time AWGN channel,

$$
Y_{i}=X_{i}+Z_{i}, \quad i=0,1, \ldots, n-1
$$

where $\boldsymbol{X}=\left(X_{0}, \ldots, X_{n-1}\right)$ is a random channel input vector, $\boldsymbol{Z}=\left(Z_{0}, \ldots, Z_{n-1}\right)$ is a zero-mean Gaussian vector with covariance matrix $\sigma^{2} I, I$ being the $n \times n$ identity matrix, and $\boldsymbol{Z}$ is statistically independent of $\boldsymbol{X}$. In the sequel, we denote the conditional probability density function (PDF) associated with this channel by $P(\boldsymbol{y} \mid \boldsymbol{x})$, that is,

$$
P(\boldsymbol{y} \mid \boldsymbol{x})=\left(2 \pi \sigma^{2}\right)^{-n / 2} \exp \left\{-\frac{1}{2 \sigma^{2}} \sum_{i=0}^{n-1}\left(y_{i}-x_{i}\right)^{2}\right\} .
$$

It is assumed that $\boldsymbol{X}$ is uniformly distributed across a codebook, $\mathcal{C}_{n}=\{\boldsymbol{x}[0], \boldsymbol{x}[1], \ldots, \boldsymbol{x}[M-1]\}$, $\boldsymbol{x}[m] \in \mathbb{R}^{n}, m=0,1, \ldots, M-1$, with $M=e^{n R}, R$ being the coding rate in nats per channel use.

We consider the random selection of the $\operatorname{codebook} \mathcal{C}_{n}$, where all codewords are drawn independently under the PDF,

$$
Q(\boldsymbol{x})= \begin{cases}\frac{1}{\operatorname{Surf}(\sqrt{n P})} & \|\boldsymbol{x}\|^{2}=n P \\ 0 & \text { elsewhere }\end{cases}
$$

where $\operatorname{Surf}(r)$ is the surface area of the $n$-dimensional Euclidean sphere of radius $r$, and $P$ is the transmission power.

Once the codebook $\mathcal{C}_{n}$ has been drawn, it is revealed to both the encoder and decoder. We consider the stochastic likelihood decoder, which randomly selects the decoded message index $\hat{m}$ according to the generalized posterior,

$$
P_{\beta}(\hat{m}=m \mid \boldsymbol{y})=\frac{P^{\beta}(\boldsymbol{y} \mid \boldsymbol{x}[m])}{\sum_{m^{\prime}=0}^{M-1} P^{\beta}\left(\boldsymbol{y} \mid \boldsymbol{x}\left[m^{\prime}\right]\right.}=\frac{\exp \left\{\beta \boldsymbol{x}^{T}[m] \cdot \boldsymbol{y} / \sigma^{2}\right\}}{\sum_{m^{\prime}=0}^{M-1} \exp \left\{\beta \boldsymbol{x}^{T}\left[m^{\prime}\right] \cdot \boldsymbol{y} / \sigma^{2}\right\}},
$$

where $\beta>0$ is a design parameter, which we select freely, and where the second equality stems from the assumption that all codewords have the same norm (energy), as is evidenced in (3). The 
motivation for the GLD (4) was discussed extensively in earlier works, such [13] and [12] as well as references therein.

The probability of error, for a given code $\mathcal{C}_{n}$, is defined as

$$
P_{\mathrm{e}}\left(\mathcal{C}_{n}\right)=\frac{1}{M} \sum_{m=0}^{M-1} \sum_{m^{\prime} \neq m} P\left(\boldsymbol{y} \mid \boldsymbol{x}_{m}\right) \cdot P_{\beta}\left(\hat{m}=m^{\prime} \mid \boldsymbol{y}\right) .
$$

As in [12], we define the error exponent of the TRC as

$$
E_{\mathrm{trc}}(R)=\lim _{n \rightarrow \infty}\left[-\frac{\boldsymbol{E}\left\{\ln P_{\mathrm{e}}\left(\mathcal{C}_{n}\right)\right\}}{n}\right]
$$

where the expectation is w.r.t. the randomness of $\mathcal{C}_{n}$, and it is assumed that the limit exists. As can be noted, this differs from the traditional random coding error exponent,

$$
E_{\mathrm{r}}(R)=\lim _{n \rightarrow \infty}\left[-\frac{\ln \left[\boldsymbol{E}\left\{P_{\mathrm{e}}\left(\mathcal{C}_{n}\right)\right\}\right]}{n}\right] .
$$

Our first goal is to derive a single-letter expression for $E_{\mathrm{trc}}(R)$.

\section{Parallel Additive Gaussian Channels and the Colored Gaussian Channel}

The model of parallel additive Gaussian channels is defined similarly as in Subsection II.B.1, except that here the various Gaussian noise components $Z_{i}, i=0,1, \ldots, n-1$, may have different variances, $\sigma_{i, n}^{2}, i=0,1, \ldots, n-1$, respectively. In other words, the covariance matrix of $\boldsymbol{Z}$ is

$\operatorname{diag}\left\{\sigma_{0, n}^{2}, \sigma_{1, n}^{2}, \ldots, \sigma_{n-1, n}^{2}\right\}$. We assume that $\left\{\sigma_{i, n}^{2}, i=0,1, \ldots, n-1\right\}$ obey an asymptotic regime where there exists some function $S_{Z}\left(e^{j \omega}\right), j=\sqrt{-1}, \omega \in[-\pi, \pi)$, such that

$$
\lim _{n \rightarrow \infty} \frac{1}{n} \sum_{i=0}^{n-1} G\left(\sigma_{i, n}^{2}\right)=\frac{1}{2 \pi} \int_{0}^{2 \pi} G\left[S_{Z}\left(e^{j \omega}\right)\right] \mathrm{d} \omega
$$

for any continuous function $G: \mathbb{R}^{+} \rightarrow \mathbb{R}$. The underlying motivation of this is the eigenvalue distribution theorem [8] (see also [7]), as will be described in Subsection II.C. Accordingly, the conditional PDF associated with this channel is given by

$$
P(\boldsymbol{y} \mid \boldsymbol{x})=\prod_{i=0}^{n-1}\left(2 \pi \sigma_{i, n}^{2}\right)^{-1 / 2} \exp \left\{-\frac{1}{2 \sigma_{i, n}^{2}}\left(y_{i}-x_{i}\right)^{2}\right\} .
$$

The structure of the code will now be slightly more involved than in Subsection II.B.1. Here we consider a codebook $\mathcal{C}_{N}$ of size $M=e^{N R}$, with block length $N=n \ell, n$ being the number 
of parallel channels as before and $\ell$ is another positive integer. Specifically, we subdivide each codeword $\boldsymbol{x}[m] \in \mathcal{C}_{N}$ of length $N$ into $n$ non-overlapping segments, each of length $\ell$, i.e., $\boldsymbol{x}[m]=$ $\left(\boldsymbol{x}_{0}[m], \boldsymbol{x}_{1}[m], \ldots, \boldsymbol{x}_{n-1}[m]\right)$, where $\boldsymbol{x}_{i}[m]=\left(x_{i \ell}[m], x_{i \ell+1}[m], \ldots, x_{(i+1) \ell-1}[m]\right), i=0,1, \ldots, n-1$. For every $i$, the segment $\boldsymbol{x}_{i}[m]$ has norm $\left\|\boldsymbol{x}_{i}[m]\right\|^{2}=\ell P_{i, n}$, and this segment is fed into the $i$-th channel component whose noise variance is $\sigma_{i, n}^{2}$. We assume that $\sum_{i=0}^{n-1} P_{i, n} \leq n P$, where $P$ is the overall power constraint. As is the case with $\left\{\sigma_{i, n}^{2}\right\}$, asymptotic convergence of the error exponent in the large $n$ limit could be expected only if there would be a well defined asymptotic regime concerning the behavior of $\left\{P_{i, n}, i=0,1, \ldots, n-1\right\}$ as $n \rightarrow \infty$. To this end, we will assume that $\left\{P_{i, n}\right\}$ are "samples" of a certain function, $S_{X}\left(e^{j \omega}\right)$, of a continuous, real-valued variable, $\omega \in[0,2 \pi)$, that is,

$$
P_{i, n}=S_{X}\left(e^{j 2 \pi i / n}\right), \quad i=0,1, \ldots, n-1 .
$$

We consider the random selection of the $\operatorname{codebook} \mathcal{C}_{N}$, where all codewords are drawn independently under the PDF,

$$
Q(\boldsymbol{x})=\prod_{i=0}^{n-1} Q_{i}\left(\boldsymbol{x}_{i}\right)
$$

where

$$
Q_{i}\left(\boldsymbol{x}_{i}\right)= \begin{cases}\frac{1}{\operatorname{Surf}\left(\sqrt{\ell P_{i, n}}\right)} & \left\|\boldsymbol{x}_{i}\right\|^{2}=\ell P_{i, n} \\ 0 & \text { elsewhere }\end{cases}
$$

Once the codebook $\mathcal{C}_{N}$ has been drawn, it is revealed to both the encoder and decoder. We consider the stochastic likelihood decoder, which randomly selects the decoded codeword $\boldsymbol{x} \in \mathcal{C}_{N}$ according to the generalized posterior,

$$
P_{\beta}(\hat{m}=m \mid \boldsymbol{y})=\frac{\exp \left\{\beta \sum_{i=0}^{n-1} \boldsymbol{x}_{i}^{T}[m] \cdot \boldsymbol{y}_{i} / \tilde{\sigma}_{i, n}^{2}\right\}}{\sum_{m^{\prime}=0}^{M-1} \exp \left\{\beta \sum_{i=0}^{n-1} \boldsymbol{x}_{i}^{T}\left[m^{\prime}\right] \cdot \boldsymbol{y}_{i} / \tilde{\sigma}_{i, n}^{2}\right\}}
$$

where $\left\{\tilde{\sigma}_{i, n}^{2}, i=0,1, \ldots, n\right\}$ are (possibly) mismatched noise variances assumed by the decoder, and $\boldsymbol{y}_{i}$ is the $i$-th segment of $\boldsymbol{y}$, that corresponds to the channel input segment $\boldsymbol{x}_{i}[m]$. Of course, if $\tilde{\sigma}_{i, n}^{2}=\sigma_{i, n}^{2}$ for all $i$ (or more generally, $\tilde{\sigma}_{i, n}^{2} \propto \sigma_{i, n}^{2}$ ) and $\beta \rightarrow \infty$, we obtain the deterministic (matched) ML decoder. Similarly as with $\left\{P_{i, n}\right\}$, it will be assumed that $\tilde{\sigma}_{i, n}^{2}=\tilde{S}_{Z}\left(e^{j 2 \pi i / n}\right)$, $i=0,1, \ldots, n-1$, for some function $\tilde{S}_{Z}\left(e^{j \omega}\right), \omega \in[0,2 \pi)$, and so, together with (10) and the eigenvalue distribution theorem, we have

$$
\lim _{n \rightarrow \infty} \frac{1}{n} \sum_{i=0}^{n-1} G\left(P_{i, n}, \sigma_{i, n}^{2}, \tilde{\sigma}_{i, n}^{2}\right)=\frac{1}{2 \pi} \int_{0}^{2 \pi} G\left[S_{X}\left(e^{j \omega}\right), S_{Z}\left(e^{j \omega}\right), \tilde{S}_{Z}\left(e^{j \omega}\right)\right] \mathrm{d} \omega
$$


for any continuous function $G$.

Finally, the probability of error and the TRC exponent are defined as in Subsection II.B.1, except that now $n$ is replaced by $N$ and the GLD of eq. (4) is replaced by the one of eq. (13). Our goal would be to derive the TRC exponent under the asymptotic regime that we have defined. The process of taking the limit of $N=n \ell \rightarrow \infty$ will be carried out in two steps: we first take the limit $\ell \rightarrow \infty$ for fixed $n$, and then take limit $n \rightarrow \infty$. In other words, the asymptotic regime corresponds to $\ell \gg n$.

\section{Background}

As mentioned in Subsection II.B.2, eq. (8) is motivated by the eigenvalue distribution theorem. Let $\left\{Z_{i}\right\}$ be a discrete-time, zero-mean stationary process with an absolutely summable autocorrelation sequence $\left\{r_{Z}(k), k=0, \pm 1, \pm 2, \ldots\right\}$ (i.e., $\left.\sum_{k}\left|r_{Z}(k)\right|<\infty\right)$, and power spectral density

$$
S_{Z}\left(e^{j \omega}\right)=\mathcal{F}\left\{r_{Z}(k)\right\}=\sum_{k=-\infty}^{\infty} r_{Z}(k) e^{-j \omega k}
$$

which is assumed strictly positive and bounded for all $\omega \in[-\pi, \pi)$. Let $R_{Z}^{n}$ be the corresponding $n \times n$ autocorrelation matrix, namely, the matrix whose $(k, l)$-th element is $r_{Z}(k-l), k, l \in$ $\{1, \ldots, n\}$. Let $\Lambda_{n}$ be the matrix whose columns are orthonormal eigenvectors of $R_{Z}^{n}$. Then by applying the linear transformation, $\tilde{Z}=\Lambda_{n}^{-1} \boldsymbol{Z}$, one diagonalizes the covariance matrix and the variances, $\left\{\sigma_{i, n}^{2}\right\}$, of the various components of $\tilde{Z}$ are equal to the respective eigenvalues of $R_{Z}^{n}$. If $\left\{r_{Z}(k)\right\}$ is absolutely summable, then according to the eigenvalue distribution theorem, eq. (8) holds true [7, Theorem 4.2] for every continuous function $G:[a, b] \rightarrow \mathbb{R}$, with $a=\operatorname{ess} \inf S_{Z}\left(e^{j \omega}\right)$ and $b=\operatorname{ess} \sup S_{Z}\left(e^{j \omega}\right)$.

Applying this setup to colored Gaussian intersymbol interference (ISI) channel model, let

$$
Y_{t}=\sum_{i=0}^{\infty} h_{i} X_{t-i}+W_{t}
$$

where $\left\{W_{t}\right\}$ is a zero-mean, stationary Gaussian process with a given spectrum $S_{W}\left(e^{j \omega}\right)$ and the linear ISI system, $H(z)=\sum_{i=0}^{\infty} h_{i} z^{-i}$, has an inverse $G(z)=1 / H(z)=\sum_{i=0}^{\infty} g_{i} z^{-i}$ such that $Z_{t}=\sum_{i=0}^{\infty} g_{i} W_{t-i}$ has a power spectral density,

$$
S_{Z}\left(e^{j \omega}\right)=S_{W}\left(e^{j \omega}\right)\left|G\left(e^{j \omega}\right)\right|^{2}=\frac{S_{W}\left(e^{j \omega}\right)}{\left|H\left(e^{j \omega}\right)\right|^{2}}
$$


whose inverse Fourier transform, $\left\{r_{Z}(k)\right\}$, is absolutely summable. Then, considering the equivalent channel model (neglecting edge effects),

$$
\tilde{Y}_{t}=X_{t}+Z_{t}
$$

we can now apply the linear transformation $\Lambda_{n}$ that diagonalizes the covariance matrix $R_{Z}^{n}$ of an $n$-block of $\boldsymbol{Z}$, and then the resulting variances, $\sigma_{i, n}^{2}$, of the transformed noise vector, are equal to the respective eigenvalues of $R_{Z}^{n}$, which in turn satisfy the eigenvalue distribution theorem in the large $n$ limit.

Operatively, the communication system works as follows: given a codeword $\boldsymbol{x}=\left(\boldsymbol{x}_{0}, \boldsymbol{x}_{1}, \ldots, \boldsymbol{x}_{n-1}\right)$, generated as described in Subsection II.B.2, we transmit $\ell$ sub-blocks, each of length $n$, where in the $k$-th sub-block $(k=0,1, \ldots, \ell-1)$, the transmitted vector is $\sum_{i=0}^{n-1} x_{i \ell+k} \psi_{i}, \psi_{i}$ being the $i^{-}$ th eigenvector of $R_{Z}^{n}$. At the receiver, we first apply the linear system $G(z)$ to $\left\{Y_{t}\right\}$ in order to obtain $\left\{\tilde{Y}_{t}\right\}$. Then, every $n$-block of $\left\{\tilde{Y}_{t}\right\}$ undergoes a bank of correlators with all eigenvectors, $\left\{\psi_{i}\right\}$, in order to retrieve noisy versions of the coefficients $\left\{x_{i n+k}\right\}_{i=0}^{n-1}$, whose noise components are uncorrelated with each other, and their variances are $\sigma_{i, n}^{2}, i=0,1, \ldots, n-1$.

Note that, in general, $\left\{\psi_{i}\right\}$ and $\left\{x_{i \ell+k}\right\}$ might be complex-valued. For the sake of simplicity, and without loss of generality, however, we will assume that they are real. This is justified by the following simple consideration. Suppose that $\psi_{i}$ is a complex eigenvector of $R_{Z}^{n}$. Since both $R_{Z}^{n}$ and the corresponding eigenvalue, $\sigma_{i, n}^{2}$, are real, then the complex conjugate, $\psi_{i}^{*}$ is also an eigenvector associated with $\sigma_{i, n}^{2}$, and therefore, so are $\boldsymbol{u}_{i}=\operatorname{Re}\left\{\psi_{i}\right\}$ and $\boldsymbol{v}_{i}=\operatorname{Im}\left\{\psi_{i}\right\}$, which are vectors in $\mathbb{R}^{n}$. Now, if $\boldsymbol{u}_{i}$ and $\boldsymbol{v}_{i}$ are not orthogonal to each other, then one can apply a simple transformation (e.g., the Gram-Schmidt projection) to represent the two-dimensional eigensubspace, spanned by $\boldsymbol{u}_{i}$ and $\boldsymbol{v}_{i}$, by two orthonormal basis vectors, which are clearly eigenvectors pertaining to $\sigma_{i, n}^{2}$ as well. Of course, eigenvectors of other eigenvalues are orthogonal to each other. For example, in the circulant approximation of $R_{Z}^{n}$ for large $n$ [7, Chapters 3,4], the matrix $\Lambda_{n}$ pertains to the discrete Fourier transform (DFT), whose complex exponential eigenvectors can be separated into real-valued sine and cosine vectors for the real and imaginary parts, which are all orthogonal to each other. 


\section{Main Results}

\section{A. The AWGN Channel}

We begin from the AWGN channel model described in Subsection II.B.1. We first define a few quantities. For a given $\sigma_{Y}^{2}>0$ and $\rho \in[-1,1]$, define

$$
g\left(\sigma_{Y}^{2}, \rho\right)=\frac{\beta \sqrt{P} \sigma_{Y} \rho}{\sigma^{2}}
$$

and

$$
\begin{aligned}
\alpha\left(R, \sigma_{Y}^{2}\right) & =\sup _{|\rho| \leq \sqrt{1-e^{-2 R}}}\left[g\left(\sigma_{Y}^{2}, \rho\right)+\frac{1}{2} \ln \left(1-\rho^{2}\right)\right]+R \\
& =w\left(\frac{\beta \sqrt{P} \sigma_{Y}}{\sigma^{2}}, R\right)+R
\end{aligned}
$$

where

$$
\begin{aligned}
w(u, R) & \triangleq \sup _{|\rho| \leq \sqrt{1-e^{-2 R}}\left[\rho \cdot u+\frac{1}{2} \ln \left(1-\rho^{2}\right)\right]} \\
& = \begin{cases}u \cdot \sqrt{1-e^{-2 R}}-R & R \leq \frac{1}{2} \ln \left(\frac{1+\sqrt{1+4 u^{2}}}{2}\right) \\
\frac{2 u^{2}}{1+\sqrt{1+4 u^{2}}}-\frac{1}{2} \ln \left(\frac{1+\sqrt{1+4 u^{2}}}{2}\right) & R \geq \frac{1}{2} \ln \left(\frac{1+\sqrt{1+4 u^{2}}}{2}\right)\end{cases}
\end{aligned}
$$

Next, for a given $\rho_{X X^{\prime}} \in[-1,1]$, define

$$
\begin{aligned}
\Gamma\left(\rho_{X X^{\prime}}\right)= & \frac{1}{2} \ln \left(2 \pi \sigma^{2}\right)+\inf \left\{\frac{1}{2 \sigma^{2}}\left(\sigma_{Y}^{2}-2 \sqrt{P} \sigma_{Y} \rho_{X Y}+P\right)-\frac{1}{2} \ln \left(2 \pi e \sigma_{Y \mid X X^{\prime}}^{2}\right)+\right. \\
& {\left.\left[\max \left\{g\left(\sigma_{Y}^{2}, \rho_{X Y}\right), \alpha\left(R, \sigma_{Y}^{2}\right)\right\}-g\left(\sigma_{Y}^{2}, \rho_{X^{\prime} Y}\right)\right]_{+}\right\} }
\end{aligned}
$$

where

$$
\sigma_{Y \mid X X^{\prime}}^{2} \triangleq \sigma_{Y}^{2}+\min _{s, t}\left\{\left(s^{2}+2 s t \rho_{X X^{\prime}}+t^{2}\right) P-2 \sqrt{P} \sigma_{Y}\left(s \rho_{X Y}+t \rho_{X^{\prime} Y}\right)\right\},
$$

and where the infimum in $(22)$ is over all $\left\{\sigma_{Y}^{2}, \rho_{X Y}, \rho_{X^{\prime} Y}\right\}$ such that the matrix

$$
\left(\begin{array}{ccc}
P & \rho_{X X^{\prime}} P & \rho_{X Y} \sqrt{P} \sigma_{Y} \\
\rho_{X X^{\prime}} P & P & \rho_{X^{\prime} Y} \sqrt{P} \sigma_{Y} \\
\rho_{X Y} \sqrt{P} \sigma_{Y} & \rho_{X^{\prime} Y} \sqrt{P} \sigma_{Y} & \sigma_{Y}^{2}
\end{array}\right)
$$

is positive semi-definite.

Our first result is the following. 
Theorem 1 For the AWGN channel model defined in Subsection II.B.1,

$$
E_{\mathrm{trc}}(R)=\inf \left\{\Gamma\left(\rho_{X X^{\prime}}\right)+\frac{1}{2} \ln \frac{1}{1-\rho_{X X^{\prime}}^{2}}\right\}-R,
$$

where the infimum is subject to the constraint $\left|\rho_{X X^{\prime}}\right| \leq \sqrt{1-e^{-4 R}}$.

The proof appears in Section V.A. The remaining part of this subsection is devoted to a discussion about Theorem 1.

The insight behind this expression of the TRC exponent is as follows. Let us imagine auxiliary random variables $X, X^{\prime}$ and $\boldsymbol{Y}$, that represent the transmitted codeword $\boldsymbol{x}[m]$, an incorrect codeword $\boldsymbol{x}\left[m^{\prime}\right]\left(m^{\prime} \neq m\right)$, and the channel output vector, $\boldsymbol{y}$, respectively. The term $\left[\max \left\{g\left(\sigma_{Y}^{2}, \rho_{X Y}\right), \alpha\left(R, \sigma_{Y}^{2}\right)\right\}-g\left(\sigma_{Y}^{2}, \rho_{X^{\prime} Y}\right)\right]_{+}$, that appears in the definition of $\Gamma\left(\rho_{X X^{\prime}}\right)$, represents the exponential rate of the probability that the GLD would select $\boldsymbol{x}\left[\mathrm{m}^{\prime}\right]$ as the decoded message given that the empirical correlation coefficient between $\boldsymbol{x}[m]$ and $\boldsymbol{x}\left[\mathrm{m}^{\prime}\right]$ is a given number, denoted $\rho_{X X^{\prime}}$. The term $\alpha\left(R, \sigma_{Y}^{2}\right)$ is interpreted as the typical exponent of the collective contribution of all incorrect codewords at the denominator of (4). As explained also in [12], the probability that $\boldsymbol{x}\left[\mathrm{m}^{\prime}\right]$ would be the decoded message given that $\boldsymbol{x}[\mathrm{m}]$ was transmitted, is of the exponential order of $\exp \left\{-n \Gamma\left(\rho_{X X^{\prime}}\right)\right\}$. Therefore, the overall error probability,

$$
\begin{aligned}
P_{\mathrm{e}}\left(\mathcal{C}_{n}\right) & =\frac{1}{M} \sum_{m} \sum_{m^{\prime} \neq m} \operatorname{Pr}\left\{\boldsymbol{x}\left[m^{\prime}\right] \text { decoded } \mid \boldsymbol{x}[m] \text { transmitted }\right\} \\
& \doteq \sum_{\rho_{X X^{\prime}}} M\left(\rho_{X X^{\prime}}\right) \exp \left\{-n\left[\Gamma\left(\rho_{X X^{\prime}}\right)+R\right]\right\}
\end{aligned}
$$

is of the exponential order of $\max _{\rho_{X X^{\prime}}} M\left(\rho_{X X^{\prime}}\right) \exp \left\{-n\left[\Gamma\left(\rho_{X X^{\prime}}\right)+R\right]\right\}$, where $M\left(\rho_{X X^{\prime}}\right)$ is the typical number of codeword pairs $\left(\boldsymbol{x}[m], \boldsymbol{x}\left[m^{\prime}\right]\right)$ whose empirical correlation coefficient is about $\rho_{X X^{\prime}}$ and the sum is over a fine grid in $(-1,1)$. Since there are about $e^{2 n R}$ codeword pairs in $\mathcal{C}_{n}$ and since the probability of event $\left\{\boldsymbol{x}^{T}[m] \cdot \boldsymbol{x}\left[m^{\prime}\right] /(n P) \approx \rho_{X X^{\prime}}\right\}$ is about $\exp \left\{-\frac{n}{2} \ln \left[1 /\left(1-\rho_{X X^{\prime}}^{2}\right)\right]\right.$, the typical value of the number $M\left(\rho_{X X^{\prime}}\right)$ is of the exponential order of $\exp \left\{n\left[2 R-\frac{1}{2} \ln \left[1 /\left(1-\rho_{X X^{\prime}}^{2}\right)\right]\right\}\right.$, whenever $2 R-\frac{1}{2} \ln \left[1 /\left(1-\rho_{X X^{\prime}}^{2}\right)>0\right.$, and is zero otherwise.

An alternative representation of $\Gamma\left(\rho_{X X^{\prime}}\right)$ is the following. Let $X, X^{\prime}$ and $Y$ be zero-mean random variables, defined as follows. The variables $X$ and $X^{\prime}$ both have variance $P$ and covariance $\boldsymbol{E}\left(X X^{\prime}\right)=\rho_{X X^{\prime}} P$. Given $X$ and $X^{\prime}$, let $Y$ be defined as $Y=a X+b X^{\prime}+V$, where $V$ is zeromean wit variance $\sigma_{V}^{2}$, and is uncorrelated to both $X$ and $X^{\prime}$. Under this representation, we 
can transform the optimization variables of $\Gamma\left(\rho_{X X^{\prime}}\right)$, from $\left\{\sigma_{Y}^{2}, \rho_{X Y}, \rho_{X^{\prime} Y}\right\}$ to $\left(a, b, \sigma_{V}^{2}\right)$, and then $\Gamma\left(\rho_{X X^{\prime}}\right)$ becomes

$$
\begin{aligned}
\Gamma\left(\rho_{X X^{\prime}}\right)= & \frac{1}{2} \ln \left(2 \pi \sigma^{2}\right)+\inf _{a, b, \sigma_{V}^{2}}\left(\frac{1}{2 \sigma^{2}}\left(\left[(a-1)^{2}+2 \rho_{X X^{\prime}}(a-1) b+b^{2}\right] P+\sigma_{V}^{2}\right)-\frac{1}{2} \ln \left(2 \pi e \sigma_{V}^{2}\right)+\right. \\
& {\left.\left[\max \left\{\frac{\beta}{\sigma^{2}}\left(a+\rho_{X X^{\prime}} b\right) P, \alpha\left(R,\left[a^{2}+2 \rho_{X X^{\prime}} a b+b^{2}\right] P+\sigma_{V}^{2}\right)\right\}-\frac{\beta}{\sigma^{2}}\left(\rho_{X X^{\prime}} a+b\right) P\right]_{+}\right), }
\end{aligned}
$$

where the infimum is over $\left(a, b, \sigma_{V}^{2}\right) \in \mathbb{R}^{2} \times \mathbb{R}^{+}$.

We observe that by eliminating the term $\alpha\left(R,\left[a^{2}+2 \rho_{X X^{\prime}} a b+b^{2}\right] P+\sigma_{V}^{2}\right)$, the expression of $\Gamma\left(\rho_{X X^{\prime}}\right)$ simplifies dramatically, and hence also the expression of the TRC exponent. As we show next, in this case, all the minimizations can be carried out in closed form. This elimination of the term $\alpha(R, \cdot)$ yields a lower bound to the TRC exponent, which corresponds to a union bound of the pairwise error events, $\left\{m \rightarrow m^{\prime}\right\}$, and which is tight at a certain range of low rates. Let us define then

$$
\begin{aligned}
& \Gamma_{L}\left(\rho_{X X^{\prime}}\right) \triangleq \frac{1}{2} \ln \left(2 \pi \sigma^{2}\right)+\inf _{a, b, \sigma_{V}^{2}}\left[\frac{1}{2 \sigma^{2}}\left(\left[(a-1)^{2}+2 \rho_{X X^{\prime}}(a-1) b+b^{2}\right] P+\sigma_{V}^{2}\right)-\right. \\
& \left.\left.\frac{1}{2} \ln \left(2 \pi e \sigma_{V}^{2}\right)+\frac{\beta P}{\sigma^{2}}\left[a+\rho_{X X^{\prime}} b-\rho_{X X^{\prime}} a-b\right)\right]_{+}\right] \\
& =\frac{1}{2} \ln \left(2 \pi \sigma^{2}\right)+\inf _{a, b, \sigma_{V}^{2}}\left[\frac{1}{2 \sigma^{2}}\left(\left[(a-1)^{2}+2 \rho_{X X^{\prime}}(a-1) b+b^{2}\right] P+\sigma_{V}^{2}\right)-\right. \\
& \left.\frac{1}{2} \ln \left(2 \pi e \sigma_{V}^{2}\right)+\frac{\beta P}{\sigma^{2}}\left(1-\rho_{X X^{\prime}}\right)[a-b]_{+}\right] \\
& =\frac{1}{2} \ln \left(2 \pi \sigma^{2}\right)+\inf _{a, b, \sigma_{V}^{2}} \sup _{0 \leq \lambda \leq \beta}\left[\frac{1}{2 \sigma^{2}}\left(\left[(a-1)^{2}+2 \rho_{X X^{\prime}}(a-1) b+b^{2}\right] P+\sigma_{V}^{2}\right)-\right. \\
& \left.\frac{1}{2} \ln \left(2 \pi e \sigma_{V}^{2}\right)+\frac{\lambda P}{\sigma^{2}} \cdot\left(1-\rho_{X X^{\prime}}\right)(a-b)\right] \\
& \stackrel{(\mathrm{a})}{=} \frac{1}{2} \ln \left(2 \pi \sigma^{2}\right)+\sup _{0 \leq \lambda \leq \beta} \inf _{a, b, \sigma_{V}^{2}}\left[\frac{1}{2 \sigma^{2}}\left(\left[(a-1)^{2}+2 \rho_{X X^{\prime}}(a-1) b+b^{2}\right] P+\sigma_{V}^{2}\right)-\right. \\
& \left.\frac{1}{2} \ln \left(2 \pi e \sigma_{V}^{2}\right)+\frac{\lambda P}{\sigma^{2}}\left(1-\rho_{X X^{\prime}}\right)(a-b)\right] \\
& \stackrel{(b)}{=} \sup _{0 \leq \lambda \leq \beta} \inf _{a, b}\left[\frac{P}{2 \sigma^{2}} \cdot\left[(a-1)^{2}+2 \rho_{X X^{\prime}}(a-1) b+b^{2}\right]+\right. \\
& \left.\frac{\lambda P}{\sigma^{2}}\left(1-\rho_{X X^{\prime}}\right)(a-b)\right] \\
& =\sup _{0 \leq \lambda \leq \beta} \frac{P}{\sigma^{2}} \cdot \lambda(1-\lambda)\left(1-\rho_{X X^{\prime}}\right) \\
& =\frac{P}{\sigma^{2}} \cdot \hat{\beta}(1-\hat{\beta}) \cdot\left(1-\rho_{X X^{\prime}}\right),
\end{aligned}
$$


where $\hat{\beta}=\min \left\{\beta, \frac{1}{2}\right\}$. Here, the passage (a) is allowed by the minimax theorem as the objective is convex in $\left(a, b, \sigma_{V}^{2}\right)$ and affine (and hence concave) in $\lambda$ and passage (b) is by the simple fact that the optimal $\sigma_{V}^{2}$ turns out to be equal to $\sigma^{2}$. Thus, for $\beta \geq \frac{1}{2}$,

$$
\Gamma_{L}\left(\rho_{X X^{\prime}}\right)=\frac{P}{4 \sigma^{2}} \cdot\left(1-\rho_{X X^{\prime}}\right) \triangleq \frac{\mathrm{snr}}{4} \cdot\left(1-\rho_{X X^{\prime}}\right) .
$$

Finally,

$$
\begin{aligned}
E_{\mathrm{trc}}(R) & \geq E_{\mathrm{trc}}^{-}(R) \\
& \triangleq \quad \inf _{\left|\rho_{X X^{\prime}}\right| \leq \sqrt{1-e^{-4 R}}\left[\frac{\mathrm{snr}}{4} \cdot\left(1-\rho_{X X^{\prime}}\right)-\frac{1}{2} \ln \left(1-\rho_{X X^{\prime}}^{2}\right)-R\right]} \\
& =\frac{\operatorname{snr}}{4}-w\left(\frac{\mathrm{snr}}{4}, 2 R\right)-R \\
& = \begin{cases}\frac{\operatorname{snr}}{4}\left(1-\sqrt{1-e^{-4 R}}\right)+R & R \leq R_{*} \\
\frac{\operatorname{snr}}{4}-\frac{\mathrm{snr}^{2} / 8}{1+\sqrt{1+\mathrm{snr}^{2} / 4}}+\frac{1}{2} \ln \left(\frac{1+\sqrt{1+\mathrm{snr}^{2} / 4}}{2}\right)-R & R \geq R_{*}\end{cases}
\end{aligned}
$$

where

$$
R_{*}=\frac{1}{4} \ln \left(\frac{1+\sqrt{1+\mathrm{snr}^{2} / 4}}{2}\right) .
$$

The lower bound, $E_{\mathrm{trc}}^{-}(R)$, has a non-affine convex part, starting with slope $-\infty$ at rate $R=0$, and ending with slope -1 at rate $R_{*}$, and so, it is tangential to the straight-line part that starts at rate $R_{*}$. The point $R=R_{*}$ exhibits a glassy phase transition [11, Chap. 6], [14, Chapters 5,6] in the behavior of $E_{\mathrm{trc}}^{-}(R)$ : for $R \leq R_{*}$, the error probability (see second line of eq. (26)) is dominated by a sub-exponential number of incorrect codewords at Euclidean distance $d=\sqrt{2 n P\left(1-\sqrt{1-e^{-4 R}}\right)}$ from the transmitted codeword, whereas for $R>R_{*}$, there are exponentially $\exp \left\{n\left[2 R-\frac{1}{2} \ln \frac{1}{1-\rho_{*}^{2}}\right]\right\}=\exp \left\{2 n\left(R-R_{*}\right)\right\}$ dominant codewords at Euclidean distance $d=\sqrt{2 n P\left(1-\rho^{*}\right)}$, where $\rho^{*}=\operatorname{snr} /\left[2\left(1+\sqrt{1+\operatorname{snr}^{2} / 4}\right)\right]$. The straight-line part of eq. (29) can readily be recognized as $R_{0}-R$, which is exactly the straight-line part of the random coding error exponent below the critical rate. Thus, the TRC exponent exceeds the random coding exponent at least in the range $R \in\left[0, R_{*}\right]$. Obviously, for $R \geq R_{\text {crit }} \geq R_{*}$, the exact TRC exponent must coincide with the random coding exponent, as the TRC exponent is sandwiched between the random coding exponent and the sphere-packing exponent, which in turn coincide for $R \geq R_{\text {crit }}$. Thus, the interesting range to explore the behavior of the TRC exponent is the range of low rates.

At the low-rate extreme, it is readily observed that the lower bound (29) yields $E_{\mathrm{trc}}(0) \geq \mathrm{snr} / 4$, which must be, in fact, an equality, since it coincides with the minimum-distance upper bound on 
the best zero-rate achievable error exponent. In this context, a natural question that arises is the following: what is the range of low rates where the above derived lower bound to the TRC exponent is tight, i.e., $E_{\mathrm{trc}}^{-}(R)=E_{\mathrm{trc}}(R)$ ? In other words, at what range of rates, the union bound of the pairwise error probabilities is of the same exponential order as the exact TRC exponent? We will propose an answer to this question in more generality in the sequel, where we consider the colored Gaussian noise model.

\section{B. Parallel Additive Gaussian Channels and the Colored Gaussian Channel}

We next move on to handle the model of independent parallel Gaussian channels, which will then be used pass to the colored Gaussian channel, as described above. Similarly as in the case of the AWGN channel, considered in Subsection III.A, here too, the elimination of the term $\alpha(R, \cdot)$ contributes dramatically to the simplification of the ultimate TRC exponent formula (lower bound). Moreover, without it, the resulting expression would be associated with a very complicated calculus of variations. Also, as before, this simplification comes at no loss of tightness at some range of low rates, as will be shown in the sequel.

Consider the setup defined in Subsection II.B.2, and to avoid cumbersome notation, we hence-

forth omit the subscript $n$ of $P_{i, n}, \sigma_{i, n}^{2}$, and $\tilde{\sigma}_{i, n}^{2}$, and denote them instead by $P_{i}, \sigma_{i}^{2}$, and $\tilde{\sigma}_{i}^{2}$, respectively. We will also use the notation $\mu_{i}=\sigma_{i}^{2} / \tilde{\sigma}_{i}^{2}$, and $\operatorname{snr}_{i}=P_{i} / \sigma_{i}^{2}$. For a given $\lambda \geq 0$ and $\theta \geq 1$, let us define

$$
A\left(\operatorname{snr}_{i}, \mu_{i}, \lambda, \theta\right) \triangleq \inf _{|\rho|<1}\left\{\lambda \mu_{i}\left(1-\lambda \mu_{i}\right) \operatorname{snr}_{i}(1-\rho)+\frac{\theta}{2} \ln \frac{1}{1-\rho^{2}}\right\}
$$

More explicitly, denoting

$$
S_{i}=\lambda \mu_{i}\left(1-\lambda \mu_{i}\right) \operatorname{snr}_{i}
$$

the minimizing $\rho$ is given by

$$
\rho_{i}^{*}=\frac{\sqrt{\theta^{2}+4 S_{i}^{2}}-\theta}{2 S_{i}}=\frac{2 S_{i}}{\sqrt{\theta^{2}+4 S_{i}^{2}}+\theta}
$$

and then

$$
A\left(\operatorname{snr}_{i}, \mu_{i}, \lambda, \theta\right)=S_{i}\left(1-\frac{2 S_{i}}{\sqrt{\theta^{2}+4 S_{i}^{2}}+\theta}\right)-\frac{\theta}{2} \ln \left[\frac{2 \theta}{\sqrt{\theta^{2}+4 S_{i}^{2}}+\theta}\right]
$$


Our first main result in this subsection is provided by the following theorem, whose proof appears in Section V.B.

Theorem 2 For the model of the parallel Gaussian channels described in Subsection II.B.2, let $\ell \rightarrow \infty$ while $n$ is kept fixed. Then,

$$
E_{\mathrm{trc}}(R) \geq \sup _{\theta \geq 1} \sup _{0 \leq \lambda \leq \beta}\left\{\frac{1}{n} \sum_{i=0}^{n-1} A\left(s n r_{i}, \mu_{i}, \lambda, \theta\right)-(2 \theta-1) R\right\} .
$$

Let $S_{X}(\omega), S_{Z}(\omega)$, and $\tilde{S}_{Z}(\omega)$ be defined as in Subsection II.B.2, and define $\operatorname{snr}(\omega)=S_{X}(\omega) / S_{Z}(\omega)$ and $\mu(\omega)=S_{Z}(\omega) / \tilde{S}_{Z}(\omega)$. The following corollary follows from Theorem 2 using the eigenvalue distribution theorem, by taking the limit $n \rightarrow \infty$.

Corollary 1 For the colored Gaussian channel described in Subsection II.B.2,

$$
E_{\mathrm{trc}}(R) \geq E_{\mathrm{trc}}^{-}(R) \triangleq \sup _{\theta \geq 1} \sup _{0 \leq \lambda \leq \beta}\left\{\frac{1}{2 \pi} \int_{0}^{2 \pi} A[\operatorname{snr}(\omega), \mu(\omega), \lambda, \theta] d \omega-(2 \theta-1) R\right\} .
$$

Referring to Corollary 1, let us denote

$$
B(\theta)=\sup _{0 \leq \lambda \leq \beta} \frac{1}{2 \pi} \int_{0}^{2 \pi} A[\operatorname{snr}(\omega), \mu(\omega), \lambda, \theta] \mathrm{d} \omega,
$$

so that

$$
E_{\text {trc }}^{-}(R)=\sup _{\theta \geq 1}[B(\theta)-(2 \theta-1) R] .
$$

A few comments are in order concerning these results.

1. The matched case. Note that in the matched case $\left(\mu_{i} \equiv 1\right.$ for parallel channels, or $\mu(\omega) \equiv 1$ for the colored channel), the optimal value ${ }^{2}$ of $\lambda$ is $\hat{\beta}=\min \left\{\beta, \frac{1}{2}\right\}$. This simplifies the formula in the sense that it remains to maximize (numerically) over one parameter only - the parameter $\theta$. It also implies that for any $\beta \geq \frac{1}{2}$, the GLD is as good as the (deterministic) ML decoder in terms of (our lower bound to) the TRC exponent.

2. General properties of the TRC exponent function. The behavior of the function $E_{\mathrm{trc}}^{-}(R)$ is similar to the that of the AWGN case. We first observe that $E_{\operatorname{trc}}^{-}(0)=\sup _{\theta \geq 1} B(\theta)=\lim _{\theta \rightarrow \infty} B(\theta)$

\footnotetext{
${ }^{2}$ Of course, any positive constant $c$, for which $\mu_{i} \equiv c$ (or $\left.\mu(\omega) \equiv c\right)$, is also associated with matched decoding, but the optimization over $\lambda$ would absorb such a constant.
} 
since $B(\theta)$ is a monotonically non-decreasing function. For low positive rates, $E_{\mathrm{trc}}^{-}(R)$ is a convex curve, with an initial slope of $-\infty$. In this range, it can be represented parametrically (with a slight abuse of notation) by the pair of equations,

$$
\begin{aligned}
R(\theta) & =\frac{1}{2} \cdot \frac{\mathrm{d} B(\theta)}{\mathrm{d} \theta} \triangleq \frac{B^{\prime}(\theta)}{2} \\
E_{\mathrm{trc}}^{-}(\theta) & =B(\theta)-(2 \theta-1) R(\theta),
\end{aligned}
$$

where $\theta$ exhausts the range $[1, \infty)$. Since $B(\theta)$ is non-decreasing and concave (because it is obtained as the minimum over affine functions of $\theta$ ), then as $R$ increases, the negative slope of $E_{\text {trc }}(R)$ becomes milder: it is given by $-\left(2 \theta_{R}-1\right)$, where $\theta_{R}$ is the solution $\theta$ to the equation $B^{\prime}(\theta)=2 R$. Since $B^{\prime}(\theta)$ is a decreasing function (due to the concavity of $B(\theta)$ ), then so is $\theta_{R}$. The curvy part of $E_{\mathrm{trc}}^{-}(R)$ ends at the point where $\theta_{R}=1$. This happens at rate $R_{*}=B^{\prime}(1) / 2$. For $R \geq R_{*}$, $E_{\mathrm{trc}}^{-}(R)=B(1)-R$, where $B(1)$ is given by

$$
B(1)=\sup _{0 \leq \lambda \leq \beta} \frac{1}{2 \pi} \int_{0}^{2 \pi} A[\operatorname{snr}(\omega), \mu(\omega), \lambda, 1] \mathrm{d} \omega .
$$

It is shown in Appendix B that this expression coincides also with $R_{0}$, the zero-rate random coding exponent, and so, in the range of rates between $R_{*}$ and $R_{\text {crit }}$, our lower bound to the TRC exponent coincides with the classical random coding exponent.

To calculate $R_{*}$, we have

$$
\begin{aligned}
R_{*} & =\frac{1}{2} \cdot \frac{\partial}{\partial \theta}\left[\frac{1}{2 \pi} \int_{0}^{2 \pi} A[\operatorname{snr}(\omega), \mu(\omega), \lambda, \theta] \mathrm{d} \omega\right]_{\theta=1} \\
& =\frac{1}{4 \pi} \int_{0}^{2 \pi}\left(\frac{\partial}{\partial \theta} A[\operatorname{snr}(\omega), \mu(\omega), \lambda, \theta]\right)_{\theta=1} \mathrm{~d} \omega \\
& =\frac{1}{4 \pi} \int_{0}^{2 \pi} \frac{\partial}{\partial \theta}\left[\lambda \mu(\omega)[1-\lambda \mu(\omega)] \operatorname{snr}(\omega)\left(1-\rho_{\theta}\right)+\frac{\theta}{2} \ln \frac{1}{1-\rho_{\theta}^{2}}\right]_{\theta=1} \mathrm{~d} \omega \\
& \stackrel{(a)}{=} \frac{1}{4 \pi} \int_{0}^{2 \pi} \frac{1}{2} \ln \frac{1}{1-\rho_{1}^{2}} \mathrm{~d} \omega \\
& =\frac{1}{8 \pi} \int_{0}^{2 \pi} \ln \left[\frac{1+\sqrt{1+4 \lambda^{2} \mu^{2}(\omega)[1-\lambda \mu(\omega)]^{2} \operatorname{snr}^{2}(\omega)}}{2}\right] \mathrm{d} \omega
\end{aligned}
$$

where $\lambda$ is the achiever of the TRC exponent and $\rho_{\theta}$ is the optimal $\rho$ for a given $\theta$. Equality (a) is obtained by observing that upon differentiating the integrand, the internal derivative $\mathrm{d} \rho_{\theta} / \mathrm{d} \theta$ is multiplied by an expression that vanishes due to the fact that $\rho_{\theta}$ (which is also a function of $\omega$ ) is 
optimal. In the matched case $(\mu(\omega) \equiv 1$, which is ML decoding), where the optimal value of $\lambda$ is $\frac{1}{2}$, this becomes

$$
R_{*}=\frac{1}{8 \pi} \int_{0}^{2 \pi} \ln \left[\frac{1+\sqrt{1+\operatorname{snr}^{2}(\omega) / 4}}{2}\right] \mathrm{d} \omega,
$$

thus recovering the expression (30) of $R_{*}$ of the AWGN channel as a special case. Obviously, $R_{\text {crit }}$ must be larger than $R_{*}$, otherwise, the TRC exponent would exceed the sphere-packing exponent along the range $\left[R_{\text {crit }}, R_{*}\right]$, which is a clear contradiction.

3. Tightness at low rates. Recall that in our analysis, both here and in the AWGN channel case, we have ignored the term $\alpha(R, \cdot)$ that designates the contribution of all the incorrect codewords in the posterior of the GLD (see eqs. (4) and (13)). It turns out that at least for deterministic mismatched decoding at low rates, this simplification comes at no cost in the exponential tightness. More precisely, there is an interval of low rates $\left[0, R_{\mathrm{t}}\right]$, where $E_{\mathrm{trc}}^{-}(R)=E_{\mathrm{trc}}(R)$. In Appendix C, we derive a non-trivial lower bound to $R_{\mathrm{t}}$.

4. The zero-rate TRC exponent. For $R=0$, we have

$$
\begin{aligned}
E_{\mathrm{trc}}^{-}(0) & =\sup _{\theta \geq 1} \sup _{0 \leq \lambda \leq \beta} \frac{1}{2 \pi} \int_{0}^{2 \pi} A[\operatorname{snr}(\omega), \mu(\omega), \lambda, \theta] \mathrm{d} \omega \\
& =\sup _{0 \leq \lambda \leq \beta} \lim _{\theta \rightarrow \infty} \frac{1}{2 \pi} \int_{0}^{2 \pi} A[\operatorname{snr}(\omega), \mu(\omega), \lambda, \theta] \mathrm{d} \omega \\
& =\sup _{0 \leq \lambda \leq \beta} \frac{1}{2 \pi} \int_{0}^{2 \pi} \lambda \mu(\omega)[1-\lambda \mu(\omega)] \operatorname{snr}(\omega) \mathrm{d} \omega \\
& =\sup _{0 \leq \lambda \leq \beta}\left\{\lambda \cdot \frac{1}{2 \pi} \int_{0}^{2 \pi} \mu(\omega) \cdot \operatorname{snr}(\omega) \mathrm{d} \omega-\lambda^{2} \cdot \frac{1}{2 \pi} \int_{0}^{2 \pi} \mu^{2}(\omega) \cdot \operatorname{snr}(\omega) \mathrm{d} \omega\right\}
\end{aligned}
$$

Now, if

$$
\beta<\frac{\int_{0}^{2 \pi} \mu(\omega) \cdot \operatorname{snr}(\omega) \mathrm{d} \omega}{2 \int_{0}^{2 \pi} \mu^{2}(\omega) \cdot \operatorname{snr}(\omega) \mathrm{d} \omega}
$$

then

$$
E_{\text {trc }}^{-}(0)=\beta \cdot \frac{1}{2 \pi} \int_{0}^{2 \pi} \mu(\omega) \cdot \operatorname{snr}(\omega) \mathrm{d} \omega-\beta^{2} \cdot \frac{1}{2 \pi} \int_{0}^{2 \pi} \mu^{2}(\omega) \cdot \operatorname{snr}(\omega) \mathrm{d} \omega .
$$

Otherwise, the optimal $\lambda$ is given by

$$
\lambda^{*}=\frac{\int_{0}^{2 \pi} \mu(\omega) \cdot \operatorname{snr}(\omega) \mathrm{d} \omega}{2 \int_{0}^{2 \pi} \mu^{2}(\omega) \cdot \operatorname{snr}(\omega) \mathrm{d} \omega}
$$


and then

$$
E_{\mathrm{trc}}^{-}(0)=\frac{1}{8 \pi} \cdot \frac{\left[\int_{0}^{2 \pi} \mu(\omega) \cdot \operatorname{snr}(\omega) \mathrm{d} \omega\right]^{2}}{\int_{0}^{2 \pi} \mu^{2}(\omega) \cdot \operatorname{snr}(\omega) \mathrm{d} \omega} .
$$

Observe that by the Cauchy-Shwartz inequality

$$
\begin{aligned}
& \frac{1}{8 \pi} \cdot \frac{\left[\int_{0}^{2 \pi} \mu(\omega) \cdot \operatorname{snr}(\omega) \mathrm{d} \omega\right]^{2}}{\int_{0}^{2 \pi} \mu^{2}(\omega) \cdot \operatorname{snr}(\omega) \mathrm{d} \omega} \\
= & \frac{1}{8 \pi} \cdot \frac{\left[\int_{0}^{2 \pi} \sqrt{\mu^{2}(\omega) \cdot \operatorname{snr}(\omega)} \cdot \sqrt{\operatorname{snr}(\omega)} \mathrm{d} \omega\right]^{2}}{\int_{0}^{2 \pi} \mu^{2}(\omega) \cdot \operatorname{snr}(\omega) \mathrm{d} \omega} \\
\leq & \frac{1}{8 \pi} \cdot \frac{\int_{0}^{2 \pi} \mu^{2}(\omega) \cdot \operatorname{snr}(\omega) \mathrm{d} \omega \cdot \int_{0}^{2 \pi} \operatorname{snr}(\omega) \mathrm{d} \omega}{\int_{0}^{2 \pi} \mu^{2}(\omega) \cdot \operatorname{snr}(\omega) \mathrm{d} \omega} \\
= & \frac{1}{8 \pi} \cdot \int_{0}^{2 \pi} \operatorname{snr}(\omega) \mathrm{d} \omega
\end{aligned}
$$

with equality iff $\mu(\omega)=$ const. for almost every $\omega$, which is the matched case.

5. The continuous-time colored Gaussian channel. A very similar analysis applies to the continuous-time colored Gaussian channel. Here, we begin from $\ell$ non-overlapping frames, each of length $T_{0}$ seconds. The resulting expression of $\log P_{\mathrm{e}}\left(C_{n}\right)$, using the very same ideas, would be of the general form $\ell \sup \sum_{i} G\left[\lambda_{i}\left(T_{0}\right)\right]$, where $\lambda_{i}\left(T_{0}\right)$ are the eigenvalues pertaining to the noise autocorrelation function (see [6, Chap. 8]). But

$$
\ell \cdot \sup \sum_{i} G\left[\lambda_{i}\left(T_{0}\right)\right]=\ell T_{0} \cdot \sup \frac{1}{T_{0}} \sum_{i} G\left[\lambda_{i}\left(T_{0}\right)\right]
$$

which for large $T_{0}$, behaves like

$$
\ell T_{0} \cdot \sup \frac{1}{2 \pi} \int_{-\infty}^{+\infty} G\left[S_{Z}(\omega)\right] \mathrm{d} \omega=T \cdot \sup \frac{1}{2 \pi} \int_{-\infty}^{+\infty} G\left[S_{Z}(\omega)\right] \mathrm{d} \omega
$$

where $T=\ell T_{0}$ is the overall duration of all $\ell$ frames of length $T_{0}$. Thus, in the continuous-time case, we obtain exactly the same error exponent formula, except for two differences: (i) $\omega$ is now analog frequency (in units of radians per second, rather than just radians), and the range of all frequency-domain integrals is from $-\infty$ to $+\infty$, rather than from 0 to $2 \pi$. (ii) The exponent is in terms of the duration $T$, rather than the integer $N$, namely, $P_{\mathrm{e}}\left(\mathcal{C}_{n}\right) \doteq e^{-E T}$ rather than $P_{\mathrm{e}}\left(\mathcal{C}_{n}\right) \doteq e^{-E n}$. 


\section{Water-Pouring Optimization of the Input Power Spectrum}

A natural question that always arises in the context of parallel Gaussian channels and the colored Gaussian channel is the question of the optimal input spectrum. This section is devoted to address this question in the context of the TRC exponent.

The function $A(\operatorname{snr}, \mu, \lambda, \theta)$ is concave in snr (as it is the minimization of affine functions of snr over the parameter $\rho$ ), and considering first, the parallel Gaussian channels, we would like to maximize $\sum_{i=0}^{n-1} A\left(\operatorname{snr}_{i}, \mu_{i}, \lambda, \theta\right)$ subject to the constraints, $\sum_{i=0}^{n-1} \sigma_{i}^{2} \operatorname{snr}_{i} \leq n P$ and $\operatorname{snr}_{i} \geq 0$ for all $i$. This amounts to the Lagrangian,

$$
\sum_{i=0}^{n-1} A\left(\operatorname{snr}_{i}, \mu_{i}, \lambda, \theta\right)+\xi\left(n P-\sum_{i=0}^{n-1} \sigma_{i}^{2} \operatorname{snr}_{i}\right)+\sum_{i=0}^{n-1} \nu_{i} \operatorname{snr}_{i} .
$$

Denoting by $\dot{A}\left(\operatorname{snr}_{i}, \mu_{i}, \lambda, \theta\right)$ the partial derivative of $A\left(\operatorname{snr}_{i}, \mu_{i}, \lambda, \theta\right)$ w.r.t. $\operatorname{snr}_{i}$, the conditions for optimality are

$$
\dot{A}\left(\operatorname{snr}_{i}, \mu_{i}, \lambda, \theta\right) \leq \xi \sigma_{i}^{2}, \quad i=0,1, \ldots, n-1
$$

with equality whenever $\operatorname{snr}_{i}>0$. The solution is

$$
\operatorname{snr}_{i}=\left[\dot{A}^{-1}\left(\xi \sigma_{i}^{2}, \mu_{i}, \lambda, \theta\right)\right]_{+}
$$

where $\dot{A}^{-1}\left(\cdot, \mu_{i}, \lambda, \theta\right)$ is the inverse ${ }^{3}$ of $\dot{A}\left(\cdot, \mu_{i}, \lambda, \theta\right)$ and $\xi \geq \dot{A}\left(0, \mu_{i}, \lambda, \theta\right) / \sigma_{\text {min }}^{2}$ (with $\sigma_{\text {min }}^{2} \triangleq$ $\left.\min _{i:} \operatorname{snr}_{i}=0 \sigma_{i}^{2}\right)$ is chosen such that

$$
\sum_{i=0}^{n-1} \sigma_{i}^{2}\left[\dot{A}^{-1}\left(\xi \sigma_{i}^{2}, \mu_{i}, \lambda, \theta\right)\right]_{+}=n P .
$$

We can now easly pass to continuous-frequency integrals as before and obtain

$$
S_{X}(\omega)=S_{Z}(\omega) \cdot\left[\dot{A}^{-1}\left(\xi S_{Z}(\omega), \mu(\omega), \lambda, \theta\right)\right]_{+},
$$

and finally maximize the resulting error-exponent expression over $\lambda$ and $\theta$. More specifically, using the concrete form of the function $A$, we have:

$$
\begin{aligned}
A(\operatorname{snr}, \mu, \lambda, \theta) & =\min _{\rho}\left\{\lambda \mu(1-\lambda \mu) \operatorname{snr}(1-\rho)+\frac{\theta}{2} \cdot \ln \frac{1}{1-\rho^{2}}\right\} \\
& =\lambda \mu(1-\lambda \mu) \operatorname{snr} \cdot[1-\rho(\operatorname{snr})]+\frac{\vartheta}{2} \cdot \ln \frac{1}{1-\rho^{2}(\mathrm{snr})},
\end{aligned}
$$

\footnotetext{
${ }^{3}$ Since $A$ is concave in snr, its derivative monotonically decreasing and hence the inverse exists.
} 
which leads to

$$
\begin{aligned}
\dot{A}(\mathrm{snr}, \mu, \lambda, \theta) & =\lambda \mu(1-\lambda \mu)[1-\rho(\mathrm{snr})]+\left[-\lambda \mu(1-\lambda \mu) \operatorname{snr}+\frac{\theta \rho(\mathrm{snr})}{1-\rho^{2}(\mathrm{snr})}\right] \cdot \frac{\mathrm{d} \rho(\mathrm{snr})}{\mathrm{dsnr}} \\
& =\lambda \mu(1-\lambda \mu)[1-\rho(\mathrm{snr})]
\end{aligned}
$$

where the second equality is due to the fact that the expression in the square brackets, which is the derivative of $A$ w.r.t. $\rho$, must vanish for $\rho$ (snr), the optimal value of $\rho$. Thus, the optimality condition is

$$
\lambda \mu(1-\lambda \mu)\left[1-\rho\left(\operatorname{snr}_{i}\right)\right] \leq \xi \sigma_{i}^{2}
$$

with equality when $\operatorname{snr}_{i}>0$, or, equivalently,

$$
\rho\left(\operatorname{snr}_{i}\right)=\frac{2 \lambda \mu(1-\lambda \mu) \operatorname{snr}_{i} / 2}{\theta+\sqrt{\theta^{2}+4 \lambda^{2} \mu^{2}(1-\lambda \mu)^{2} \operatorname{snr}_{i}^{2}}}=\left[1-\frac{\xi \sigma_{i}^{2}}{\lambda \mu(1-\lambda \mu)}\right]_{+} .
$$

Upon solving for $\operatorname{snr}_{i}$, one obtains

$$
\operatorname{snr}_{i}=\frac{1}{\lambda \mu_{i}\left(1-\lambda \mu_{i}\right)} \cdot \frac{\theta\left[1-\xi \sigma_{i}^{2} /\left\{\lambda \mu_{i}\left(1-\lambda \mu_{i}\right)\right\}\right]_{+}}{1-\left[1-\xi \sigma_{i}^{2} /\left\{\lambda \mu_{i}\left(1-\lambda \mu_{i}\right)\right\}\right]_{+}^{2}} .
$$

Passing to the limit of the continuous frequency domain, we obtain

$$
\begin{aligned}
S_{X}(\omega) & =\frac{S_{Z}(\omega)}{\lambda \mu(\omega)[1-\lambda \mu(\omega)]} \cdot \frac{\left.\theta\left[1-\xi S_{Z}(\omega) /\{\lambda \mu(\omega)[1-\lambda \mu(\omega)])\right\}\right]_{+}}{1-\left[1-\xi S_{Z}(\omega) /\{\lambda \mu(\omega)[1-\lambda \mu(\omega)]\}\right]_{+}^{2}} \\
& = \begin{cases}\frac{\theta\left\{\lambda \mu(\omega)[1-\lambda \mu(\omega)]-\xi S_{Z}(\omega)\right\}}{2 \xi \lambda \mu(\omega)[1-\lambda \mu(\omega)]-\xi^{2} S_{Z}(\omega)} & \xi S_{Z}(\omega) \leq \lambda \mu(\omega)[1-\lambda \mu(\omega)] \\
0 & \text { elsewhere }\end{cases}
\end{aligned}
$$

or, denoting $B=1 / 4 \xi$,

$$
\begin{aligned}
S_{X}(\omega) & = \begin{cases}\frac{4 \theta B\left\{4 B \lambda \mu(\omega)[1-\lambda \mu(\omega)]-S_{Z}(\omega)\right\}}{8 B \lambda \mu(\omega)[1-\lambda \mu(\omega)]-S_{Z}(\omega)} & S_{Z}(\omega) \leq 4 B \lambda \mu(\omega)[1-\lambda \mu(\omega)] \\
0 & \text { elsewhere }\end{cases} \\
& =\frac{4 \theta B\left[4 B \lambda \mu(\omega)\{1-\lambda \mu(\omega)\}-S_{Z}(\omega)\right]_{+}}{4 B \lambda \mu(\omega)[1-\lambda \mu(\omega)]+\left[4 B \lambda \mu(\omega)\{1-\lambda \mu(\omega)\}-S_{Z}(\omega)\right]_{+}}
\end{aligned}
$$

where $B$ is chosen such that

$$
\frac{1}{2 \pi} \int_{0}^{2 \pi} \frac{4 B\left[4 B \lambda \mu(\omega)\{1-\lambda \mu(\omega)\}-S_{Z}(\omega)\right]_{+}}{4 B \lambda \mu(\omega)[1-\lambda \mu(\omega)]+\left[4 B \lambda \mu(\omega)\{1-\lambda \mu(\omega)\}-S_{Z}(\omega)\right]_{+}} \cdot \mathrm{d} \omega=\frac{P}{\theta} .
$$

Note that the optimum input spectrum depends on $R$, via the variable $\theta$, whose optimal value depends on $R$. When $\theta \rightarrow \infty(R \rightarrow 0)$, the r.h.s. goes to zero, and $B$ must be chosen just slightly 
above $\min _{\omega} S_{Z}(\omega) /\{\lambda \mu(\omega)[1-\lambda \mu(\omega)]\}$, in order to comply with the power constraint. This means that $S_{X}(\omega)$ would tend to concentrate all the power at the frequency $\omega^{*}$, which achieves this minimum.

In the matched case, where $\lambda \mu(\omega) \equiv 1 / 2$, we obtain

$$
S_{X}(\omega)=\frac{4 \theta B\left[B-S_{Z}(\omega)\right]_{+}}{B+\left[B-S_{Z}(\omega)\right]_{+}} .
$$

This optimal spectral power distribution is identical to that of the ordinary expurgated exponent for parallel additive Gaussian channel (see [6, eq. (7.5.51), p. 352]). This should not be very surprising as the expurgated exponent and the TRC exponent are closely related [1], [12].

\section{Proofs}

For the proofs, we will need some additional notation and a few preliminary facts.

The empirical variance of a sequence $\boldsymbol{x} \in \mathbb{R}^{n}$ is defined as

$$
\hat{\sigma}_{\boldsymbol{x}}^{2}=\frac{1}{n} \sum_{i=1}^{n} x_{i}^{2}=\frac{\|\boldsymbol{x}\|^{2}}{n}
$$

For a given $\epsilon>0$, the Gaussian type class of $\boldsymbol{x} \in \mathbb{R}^{n}$ with tolerance $\epsilon$, will be defined as

$$
\mathcal{T}_{\epsilon}(\boldsymbol{x})=\left\{\boldsymbol{x}^{\prime}:\left|\hat{\sigma}_{\boldsymbol{x}^{\prime}}^{2}-\hat{\sigma}_{\boldsymbol{x}}^{2}\right| \leq \epsilon\right\}
$$

The differential entropy associated with $\hat{\sigma}_{\boldsymbol{x}}^{2}$, which is the Gaussian empirical entropy of $\boldsymbol{x}$, will be defined as

$$
\hat{h}_{\boldsymbol{x}}(X)=\frac{1}{2} \ln \left(2 \pi e \hat{\sigma}_{\boldsymbol{x}}^{2}\right) .
$$

Similar conventions will apply to conditional empirical conditional types and empirical conditional Gaussian differential entropies and mutual informations. The empirical covariance of $(\boldsymbol{x}, \boldsymbol{y}) \in$ $\mathbb{R}^{n} \times \mathbb{R}^{n}$ will be defined as

$$
\hat{C} \boldsymbol{x} \boldsymbol{y}=\frac{1}{n} \sum_{i=1}^{n} x_{i} y_{i}=\frac{\boldsymbol{x}^{T} \boldsymbol{y}}{n},
$$

The empirical correlation coefficient of $(\boldsymbol{x}, \boldsymbol{y}) \in \mathbb{R}^{n} \times \mathbb{R}^{n}$ will be defined as

$$
\hat{\rho} \boldsymbol{x} \boldsymbol{y}=\frac{\hat{C}_{\boldsymbol{x} \boldsymbol{y}}}{\hat{\sigma} \boldsymbol{x} \hat{\sigma} \boldsymbol{y}} .
$$


Accordingly, for a given $\epsilon>0$, the Gaussian conditional type class of $\boldsymbol{y} \in \mathbb{R}^{n}$ given $\boldsymbol{x} \in \mathbb{R}^{n}$, with tolerance $\epsilon$, will be defined as

$$
\mathcal{T}_{\epsilon}(\boldsymbol{y} \mid \boldsymbol{x})=\left\{\boldsymbol{y}^{\prime}:\left|\hat{\sigma}_{\boldsymbol{y}^{\prime}}^{2}-\hat{\sigma}_{\boldsymbol{y}}^{2}\right| \leq \epsilon,\left|\hat{C}_{\boldsymbol{x} \boldsymbol{y}^{\prime}}-\hat{C}_{\boldsymbol{x} \boldsymbol{y}}\right| \leq \epsilon\right\}
$$

The Gaussian empirical conditional entropy of $\boldsymbol{y}$ given $\boldsymbol{x}$ will be defined as

$$
\hat{h}_{\boldsymbol{x} \boldsymbol{y}}(Y \mid X)=\frac{1}{2} \ln \left[2 \pi e \hat{\sigma}_{\boldsymbol{y}}^{2}\left(1-\hat{\rho}_{\boldsymbol{x} \boldsymbol{y}}^{2}\right)\right]=\frac{1}{2} \ln \left[2 \pi e\left(\hat{\sigma}_{\boldsymbol{y}}^{2}-\frac{\hat{C}_{\boldsymbol{x} \boldsymbol{y}}^{2}}{\hat{\sigma}_{\boldsymbol{x}}^{2}}\right)\right],
$$

and so, the Gaussian empirical mutual information is given by

$$
\hat{I}_{\boldsymbol{x y}}(X ; Y)=\hat{h}_{\boldsymbol{y}}(Y)-\hat{h}_{\boldsymbol{x} \boldsymbol{y}}(Y \mid X)=\frac{1}{2} \ln \frac{1}{1-\hat{\rho}_{\boldsymbol{x} \boldsymbol{y}}^{2}} .
$$

Note that $\hat{h}_{\boldsymbol{x} \boldsymbol{y}}(X \mid Y)$ can also be presented as

$$
\hat{h}_{\boldsymbol{x} \boldsymbol{y}}(X \mid Y)=\frac{1}{2} \ln \left[2 \pi e \hat{\sigma}_{\boldsymbol{y} \mid \boldsymbol{x}}^{2}\right]
$$

where

$$
\hat{\sigma}_{\boldsymbol{y} \mid \boldsymbol{x}}^{2}=\min _{a \in \mathbb{R}} \frac{1}{n}\|\boldsymbol{y}-a \boldsymbol{x}\|^{2}=\hat{\sigma}_{\boldsymbol{y}}^{2}\left(1-\hat{\rho}_{\boldsymbol{x} \boldsymbol{y}}^{2}\right),
$$

and so, the notion of the Gaussian conditional empirical differential entropy can easily apply to conditioning on more than one vector. For example, given $\left(\boldsymbol{x}, \boldsymbol{x}^{\prime}, \boldsymbol{y}\right) \in\left(\mathbb{R}^{n}\right)^{3}$,

$$
\hat{h}_{\boldsymbol{x} \boldsymbol{x}^{\prime} \boldsymbol{y}}\left(Y \mid X, X^{\prime}\right)=\frac{1}{2} \ln \left[2 \pi e \hat{\sigma}_{\boldsymbol{y} \mid \boldsymbol{x} \boldsymbol{x}^{\prime}}^{2}\right],
$$

with

$$
\begin{aligned}
\hat{\sigma}_{\boldsymbol{y} \mid \boldsymbol{x} \boldsymbol{x}^{\prime}}^{2} & =\min _{a, b \in \mathbb{R}} \frac{1}{n}\left\|\boldsymbol{y}-a \boldsymbol{x}-b \boldsymbol{x}^{\prime}\right\|^{2} \\
& =\min _{a, b \in \mathbb{R}}\left\{\hat{\sigma}_{\boldsymbol{y}}^{2}-2\left(a \hat{C}_{\boldsymbol{x} \boldsymbol{y}}+b \hat{C}_{\boldsymbol{x}^{\prime} \boldsymbol{y}}\right)+\left(a^{2}+2 a b \hat{\rho} \boldsymbol{x} \boldsymbol{x}^{\prime}+b^{2}\right) P\right\}
\end{aligned}
$$

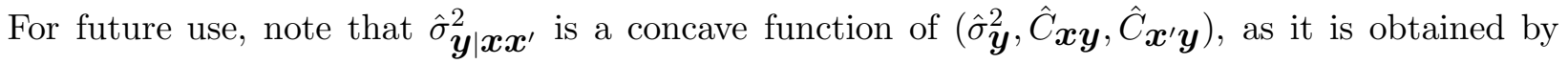
minimizing an affine function of these variables. Since the logarithmic function is monotonically

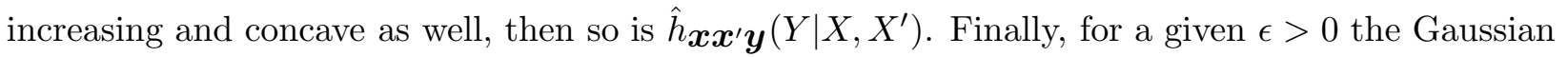
conditional type class of $\boldsymbol{y} \in \mathbb{R}^{n}$ given $\boldsymbol{x}, \boldsymbol{x}^{\prime} \in \mathbb{R}^{n}$, with tolerance $\epsilon$, will be defined as

$$
\mathcal{T}_{\epsilon}\left(\boldsymbol{y} \mid \boldsymbol{x}, \boldsymbol{x}^{\prime}\right)=\left\{\tilde{\boldsymbol{y}}:\left|\hat{\sigma}_{\tilde{\boldsymbol{y}}}^{2}-\hat{\sigma}_{\boldsymbol{y}}^{2}\right| \leq \epsilon,\left|\hat{C}_{\boldsymbol{x} \tilde{\boldsymbol{y}}}-\hat{C}_{\boldsymbol{x} \boldsymbol{y}}\right| \leq \epsilon,\left|\hat{C}_{\boldsymbol{x}^{\prime}} \tilde{\boldsymbol{y}}-\hat{C}_{\boldsymbol{x}^{\prime} \boldsymbol{y}}\right| \leq \epsilon\right\}
$$

In the sequel, we will need the following simple inequality:

$$
\operatorname{Vol}\left\{\mathcal{T}_{\epsilon}\left(\boldsymbol{y} \mid \boldsymbol{x}, \boldsymbol{x}^{\prime}\right)\right\} \leq \exp \left\{n\left[\hat{h}_{\boldsymbol{x} \boldsymbol{x}} \boldsymbol{y}\left(Y \mid X, X^{\prime}\right)+O(\epsilon)\right]\right\}
$$

which is easily proved using the same technique as in [10, Lemma 3]. 


\section{A. Proof of Theorem 1}

The proof is based on the same line of thought as in [12, Theorem 1], except that some modifications have to be carried out in order to address the continuous alphabet case considered here. As in [12], we use the identity

$$
\boldsymbol{E}\left\{\ln P_{\mathrm{e}}\left(\mathcal{C}_{n}\right)\right\}=\lim _{r \rightarrow \infty} r \cdot \ln \left(\boldsymbol{E}\left\{P_{\mathrm{e}}\left(\mathcal{C}_{n}\right)^{1 / r}\right\}\right),
$$

and so, we first focus on the calculation of $\boldsymbol{E}\left\{P_{\mathrm{e}}\left(\mathcal{C}_{n}\right)^{1 / r}\right\}$. Another simple observation (easily proved using the Chernoff bound) that will be used in the proof is that for every $E>0$, there exists a sufficiently large constant $B>0$, such that for every $m \in\{0,1, \ldots, M-1\}$,

$$
\operatorname{Pr}\left\{\|\boldsymbol{Y}\|^{2} \geq n B \text { or }\|\boldsymbol{Y}\|^{2} \leq n / B \mid \boldsymbol{x}[m] \text { transmitted }\right\} \leq e^{-n E},
$$

and so if we take $E=E_{\text {ex }}(0)$, the zero-rate expurgated exponent, we can find a constant $B$ such that the contribution of $\operatorname{Pr}\left\{\|\boldsymbol{Y}\|^{2} \leq n / B\right\}+\operatorname{Pr}\left\{\|\boldsymbol{Y}\|^{2} \geq n B\right\}$ cannot affect the error exponent $E_{\text {trc }}(R) \leq E_{\text {ex }}(0)$ at any coding rate (see also [10] for a similar argument). In other words, if the decoder would declare an error for every $\boldsymbol{y}$ whose norm is either larger than $n B$ or smaller than $n / B$, there would be no degradation in the error exponent. Consequently, it is enough to focus only on $\boldsymbol{y}$-vectors whose norms are in the range $[n / B, n B]$. Let us denote this set of vectors by $\mathcal{H}_{n}(B)$. Now,

$$
\begin{aligned}
P_{\mathrm{e}}\left(\mathcal{C}_{n}\right) & =\frac{1}{M} \sum_{m=0}^{M-1} \sum_{m^{\prime} \neq m} \int_{\mathbb{R}^{n}} P\left(\boldsymbol{y} \mid \boldsymbol{x}_{m}\right) \cdot \frac{\exp \left\{n \beta \boldsymbol{x}^{T}\left[m^{\prime}\right] \cdot \boldsymbol{y} / \sigma^{2}\right\}}{\sum_{\tilde{m}=0}^{M-1} \exp \left\{n \beta \boldsymbol{x}^{T}[\tilde{m}] \cdot \boldsymbol{y} / \sigma^{2}\right\}} \cdot \mathrm{d} \boldsymbol{y} \\
& =\frac{1}{M} \sum_{m=0}^{M-1} \sum_{m^{\prime} \neq m} \int_{\mathbb{R}^{n}} P\left(\boldsymbol{y} \mid \boldsymbol{x}_{m}\right) \cdot \frac{\exp \left\{n \beta \boldsymbol{x}^{T}\left[m^{\prime}\right] \cdot \boldsymbol{y} / \sigma^{2}\right\}}{\exp \left\{n \beta \boldsymbol{x}^{T}[m] \cdot \boldsymbol{y} / \sigma^{2}\right\}+\sum_{\tilde{m} \neq m} \exp \left\{n \beta \boldsymbol{x}^{T}[\tilde{m}] \cdot \boldsymbol{y} / \sigma^{2}\right\}} \cdot \mathrm{d} \boldsymbol{y} \\
& \doteq \frac{1}{M} \sum_{m=0}^{M-1} \sum_{m^{\prime} \neq m} \int_{\mathcal{H}_{n}(B)} P\left(\boldsymbol{y} \mid \boldsymbol{x}_{m}\right) \cdot \frac{\exp \left\{n \beta \boldsymbol{x}^{T}\left[m^{\prime}\right] \cdot \boldsymbol{y} / \sigma^{2}\right\}}{\exp \left\{n \beta \boldsymbol{x}^{T}[m] \cdot \boldsymbol{y} / \sigma^{2}\right\}+Z_{m}(\boldsymbol{y})} \cdot \mathrm{d} \boldsymbol{y},
\end{aligned}
$$

where we have defined

$$
Z_{m}(\boldsymbol{y})=\sum_{\tilde{m} \neq m} \exp \left\{n \beta \boldsymbol{x}^{T}[\tilde{m}] \cdot \boldsymbol{y} / \sigma^{2}\right\}
$$

We now argue that for every $\epsilon>0$ (see Appendix for the proof),

$$
\operatorname{Pr}\left\{\mathcal{C}_{n}: \exists 0 \leq m<M, \boldsymbol{y} \in \mathcal{H}_{n}(B): Z_{m}(\boldsymbol{y})<\exp \left\{n\left[\alpha\left(R-\epsilon, \hat{\sigma}_{\boldsymbol{y}}^{2}\right)-\delta(\epsilon)\right]\right\}\right\} \doteq e^{-n \infty}
$$


where $\delta(\epsilon)=2 \beta \sqrt{P} B \epsilon / \sigma^{2}$. We then have (neglecting $\epsilon$, which is arbitrarily small),

$$
\begin{aligned}
& \boldsymbol{E}\left\{\left[P_{\mathrm{e}}\left(\mathcal{C}_{n}\right)\right]^{1 / r}\right\} \leq \boldsymbol{E}\left(\left[\frac{1}{M} \sum_{m=0}^{M-1} \sum_{m^{\prime} \neq m} \int_{\mathcal{H}_{n}(B)} P\left(\boldsymbol{y} \mid \boldsymbol{x}_{m}\right) \times\right.\right. \\
& \left.\left.\min \left\{1, \frac{\exp \left\{n \beta \boldsymbol{x}^{T}\left[m^{\prime}\right] \boldsymbol{y} / \sigma^{2}\right\}}{\exp \left\{n \beta \boldsymbol{x}^{T}[m] \boldsymbol{y} / \sigma^{2}\right\}+\exp \left\{n \alpha\left(R, \hat{\sigma}_{\boldsymbol{y}}^{2}\right)\right\}}\right\} \mathrm{d} \boldsymbol{y}\right]^{1 / r}\right) \\
& \doteq \boldsymbol{E}\left(\left[\frac{1}{M} \sum_{m=0}^{M-1} \sum_{m^{\prime} \neq m} \int_{\mathcal{H}_{n}(B)} P\left(\boldsymbol{y} \mid \boldsymbol{x}_{m}\right) \times\right.\right. \\
& \left.\left.\min \left\{1, \frac{\exp \left\{n \beta \boldsymbol{x}^{T}\left[m^{\prime}\right] \boldsymbol{y} / \sigma^{2}\right\}}{\exp \left\{n \beta \boldsymbol{x}^{T}[m] \boldsymbol{y} / \sigma^{2}\right\}+\exp \left\{n \alpha\left(R, \hat{\sigma}_{\boldsymbol{y}}^{2}\right)\right\}}\right\} \mathrm{d} \boldsymbol{y}\right]^{1 / r}\right) \\
& \doteq \boldsymbol{E}\left(\left[\frac{1}{M} \sum_{m=0}^{M-1} \sum_{m^{\prime} \neq m} \int_{\mathcal{H}_{n}(B)} P\left(\boldsymbol{y} \mid \boldsymbol{x}_{m}\right) \times\right.\right. \\
& \left.\left.\exp \left\{-n\left[\max \left\{\frac{\beta \sqrt{P} \hat{\sigma} \boldsymbol{y}}{\sigma^{2}} \hat{\rho}_{\boldsymbol{x}[m] \boldsymbol{y}}, \alpha\left(R, \hat{\sigma}_{\boldsymbol{y}}^{2}\right)\right\}-\frac{\beta \sqrt{P} \hat{\sigma} \boldsymbol{y}}{\sigma^{2}} \hat{\rho}_{\boldsymbol{x}\left[m^{\prime}\right]}\right]_{+}\right\} \mathrm{d} \boldsymbol{y}\right]^{1 / r}\right) \text {, }
\end{aligned}
$$

where we have neglected the double-exponentially small contribution of codes that violate (82). The inner integral over $\boldsymbol{y}$ is of the form

$$
\int_{\mathcal{H}_{n}(B)}\left(2 \pi \sigma^{2}\right)^{-n / 2} \exp \left\{-\frac{\|\boldsymbol{y}-\boldsymbol{x}[m]\|^{2}}{2 \sigma^{2}}\right\} \cdot \exp \left\{-n K\left(\hat{\sigma} \boldsymbol{y}, \hat{\rho} \boldsymbol{x}[m] \boldsymbol{y}, \hat{\rho} \boldsymbol{x}\left[m^{\prime}\right] \boldsymbol{y}\right)\right\} \mathrm{d} \boldsymbol{y},
$$

where

$$
K\left(\sigma_{Y}, \rho_{X Y}, \rho_{X^{\prime} Y}\right)=\left[\max \left\{\frac{\beta \sqrt{P} \sigma_{Y}}{\sigma^{2}} \rho_{X Y}, \alpha\left(R, \sigma_{Y}^{2}\right)\right\}-\frac{\beta \sqrt{P} \sigma_{Y}}{\sigma^{2}} \rho_{X^{\prime} Y}\right]_{+} .
$$

Since $K$ is not a quadratic function, this is not a simple Gaussian integral, but its exponential order can be assessed using a Gaussian analogue of the method of types (see, e.g., the analysis in [10]). For the given two codewords, $\boldsymbol{x}[m]$ and $\boldsymbol{x}\left[m^{\prime}\right]$, we divide $\mathcal{H}_{n}(B)$ into conditional types $\left\{\mathcal{T}_{\epsilon}\left(\boldsymbol{y} \mid \boldsymbol{x}[m], \boldsymbol{x}\left[m^{\prime}\right]\right)\right\}$. The number of such conditional types is finite: since $\|\boldsymbol{y}\|^{2} \leq n B$ and $\|\boldsymbol{x}[m]\|^{2}=\left\|\boldsymbol{x}\left[m^{\prime}\right]\right\|^{2}=n P$, then $\hat{C}_{\boldsymbol{x}[m] \boldsymbol{y}}$ and $\hat{C}_{\boldsymbol{x}\left[m^{\prime}\right]} \boldsymbol{y}$ can take on values only in the interval $[-\sqrt{P B}, \sqrt{P B}]$, and so, there are no more than $(B / \epsilon) \cdot(2 \sqrt{P B} / \epsilon)^{2}=4 P B^{2} / \epsilon^{3}$ conditional types classes $\left\{\mathcal{T}_{\epsilon}\left(\boldsymbol{y} \mid \boldsymbol{x}[m], \boldsymbol{x}\left[m^{\prime}\right]\right)\right\}$ within $\mathcal{H}_{n}(B)$, resulting from a grid of step size $\epsilon$ in each of the ranges

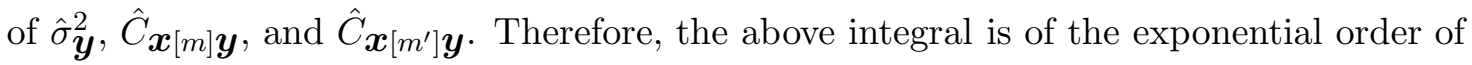

$$
\begin{aligned}
& \sup _{\boldsymbol{y} \in \mathcal{H}_{n}(B)} \operatorname{Vol}\left\{\mathcal{T}_{\epsilon}\left(\boldsymbol{y} \mid \boldsymbol{x}[m], \boldsymbol{x}\left[m^{\prime}\right]\right)\right\} \cdot\left(2 \pi \sigma^{2}\right)^{-n / 2} \exp \left\{-\frac{\|\boldsymbol{y}-\boldsymbol{x}[m]\|^{2}}{2 \sigma^{2}}\right\} \times \\
& \exp \left\{-n K\left(\hat{\sigma}_{\boldsymbol{y}}, \hat{\rho}_{\boldsymbol{x}[m] \boldsymbol{y}}, \hat{\rho}_{\left.\left.\boldsymbol{x}\left[m^{\prime}\right] \boldsymbol{y}\right)\right\}}\right.\right.
\end{aligned}
$$




$$
\begin{aligned}
\doteq & \exp \left\{-n \inf _{\boldsymbol{y}}\left[\frac{1}{2} \ln \left(2 \pi \sigma^{2}\right)+\frac{\hat{\sigma}_{\boldsymbol{y}}^{2}-2 \sqrt{P} \hat{\sigma} \boldsymbol{y}+P}{2 \sigma^{2}}-\hat{h}_{\boldsymbol{y} \mid \boldsymbol{x}[m] \boldsymbol{x}\left[m^{\prime}\right]}\left(Y \mid X, X^{\prime}\right)+\right.\right. \\
& \left.\left.K\left(\hat{\sigma} \boldsymbol{y}, \hat{\rho} \boldsymbol{x}[m] \boldsymbol{y}, \hat{\rho} \boldsymbol{x}\left[m^{\prime}\right] \boldsymbol{y}\right)\right]\right\} \\
\leq & \exp \left\{-n \inf _{\sigma_{Y}, \rho_{X Y}, \rho_{X^{\prime} Y}}\left[\frac{1}{2} \ln \left(2 \pi \sigma^{2}\right)+\frac{\sigma_{Y}^{2}-2 \sqrt{P} \sigma_{Y}+P}{2 \sigma^{2}}-\frac{1}{2} \ln \left(2 \pi e \sigma_{Y \mid X X^{\prime}}^{2}\right)+\right.\right. \\
& \left.\left.K\left(\sigma_{Y}, \rho_{X Y}, \rho_{X^{\prime} Y}\right)\right]\right\}
\end{aligned}
$$

where the infimum over $\left(\sigma_{Y}, \rho_{X Y}, \rho_{X^{\prime} Y}\right)$ is such that the matrix (24) (with $\left.\rho_{X X^{\prime}}=\hat{\rho}_{\boldsymbol{x}[m] \boldsymbol{x}\left[m^{\prime}\right]}\right)$ is positive semi-definite. Using eqs. (75) and (77), it is readily observed that this integral is of the exponential order of $e^{-n \Gamma\left(\rho \boldsymbol{x}_{[m]} \boldsymbol{x}_{\left[m^{\prime}\right]}\right)}$, where the function $\Gamma(\cdot)$ is defined in (22). Thus, we have shown that

$$
\begin{aligned}
\boldsymbol{E}\left\{\left[P_{\mathrm{e}}\left(\mathcal{C}_{n}\right)\right]^{1 / r}\right\} & \leq \boldsymbol{E}\left(\left[\frac{1}{M} \sum_{m=0}^{M-1} \sum_{m^{\prime} \neq m} \exp \left\{-n \Gamma\left(\rho_{\boldsymbol{x}[m]} \boldsymbol{x}\left[m^{\prime}\right]\right)\right\}\right]^{1 / r}\right) \\
& \leq e^{n R / r} \boldsymbol{E}\left(\sum_{\rho_{i}} M\left(\rho_{i}\right) \exp \left\{-n \Gamma\left(\rho_{i}\right)\right\}\right)^{1 / r} \\
& \leq e^{n R / r} \sum_{\rho_{i}} \boldsymbol{E}\left\{M\left(\rho_{i}\right)^{1 / r}\right\} \cdot \exp \left\{-n \Gamma\left(\rho_{i}\right) / r\right\}
\end{aligned}
$$

where $\left\{\rho_{i}\right\}$ form a fine quantization grid over the interval $(-1,1)$ and $M\left(\rho_{i}\right)$ is the number of codeword pairs $\left\{\boldsymbol{x}[m], \boldsymbol{x}\left[\mathrm{m}^{\prime}\right]\right\}$ whose empirical correlation coefficient fall in the quantization bin of $\rho_{i}$. The remaining part of the proof follows exactly the same lines as the proof of [12, Theorem 1], except that joint types $\left\{Q_{X X^{\prime}}\right\}$ (and the conditional types, $\left\{Q_{X^{\prime} \mid X}\right\}$ ) of [12] are now indexed by $\left\{\rho_{i}\right\}$ and $I(Q)$ is replaced by $\frac{1}{2} \ln \frac{1}{1-\rho_{X X^{\prime}}^{2}}$.

\section{B. Proof of Theorem 2}

The proof goes along the same lines as the proof of Theorem 1, except that there are $n$ independent copies of all elements produced from $\ell$-vectors, and so, we will outline only the main differences. Here we denote

$$
\begin{aligned}
\hat{\sigma}_{\boldsymbol{y}_{i}}^{2} & =\frac{\left\|\boldsymbol{y}_{i}\right\|^{2}}{\ell} \\
\hat{C}_{\boldsymbol{x}_{i} \boldsymbol{y}_{i}} & =\frac{\boldsymbol{x}_{i}^{T} \boldsymbol{y}_{i}}{\ell} .
\end{aligned}
$$


For a given code $\mathcal{C}_{n}$, a given transmitted codeword $\boldsymbol{x}[m]$ and a given competing codeword $\boldsymbol{x}\left[\mathrm{m}^{\prime}\right]$,

$$
\begin{aligned}
& \operatorname{Pr}\left\{m^{\prime} \text { decoded } \mid m \text { transmitted }\right\} \\
& =\int_{\mathbb{R}^{N}} P(\boldsymbol{y} \mid \boldsymbol{x}[m]) \cdot \operatorname{Pr}\left\{\hat{m}=m^{\prime} \mid \boldsymbol{y}\right\} \mathrm{d} \boldsymbol{y} \\
& \leq \int_{\mathbb{R}^{N}}\left[\prod_{i=0}^{n-1}\left(2 \pi \sigma_{i}^{2}\right)^{-\ell / 2} \exp \left\{-\left\|\boldsymbol{y}_{i}-\boldsymbol{x}_{i}[m]\right\|^{2} /\left(2 \sigma_{i}^{2}\right)\right\}\right] \times \\
& \min \left\{1, \exp \left[\beta \sum_{i=0}^{n-1}\left(\frac{\boldsymbol{x}_{i}^{T}\left[m^{\prime}\right] \boldsymbol{y}_{i}}{\tilde{\sigma}_{i}^{2}}-\frac{\boldsymbol{x}_{i}^{T}[m] \boldsymbol{y}_{i}}{\tilde{\sigma}_{i}^{2}}\right)\right]\right\} \mathrm{d} \boldsymbol{y}
\end{aligned}
$$

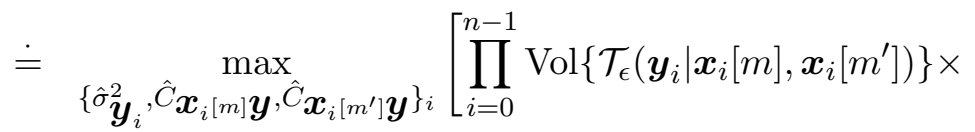

$$
\begin{aligned}
& \left.\left(2 \pi \sigma_{i}^{2}\right)^{-\ell / 2} \exp \left\{-\left\|\boldsymbol{y}_{i}-\boldsymbol{x}_{i}[m]\right\|^{2} /\left(2 \sigma_{i}^{2}\right)\right\}\right] \cdot \exp \left\{-\beta\left[\sum_{i=0}^{n-1}\left(\frac{\boldsymbol{x}_{i}^{T}[m] \boldsymbol{y}_{i}}{\tilde{\sigma}_{i}^{2}}-\frac{\boldsymbol{x}_{i}^{T}\left[m^{\prime}\right] \boldsymbol{y}_{i}}{\tilde{\sigma}_{i}^{2}}\right)\right]_{+}\right\}
\end{aligned}
$$

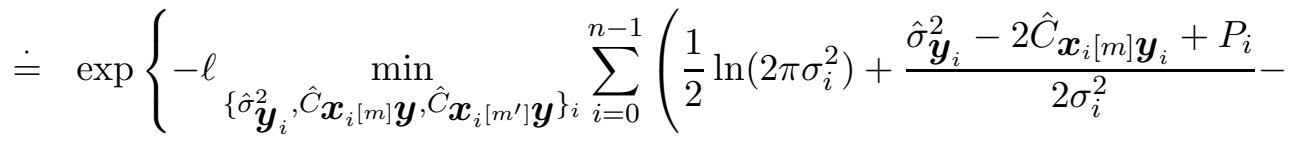

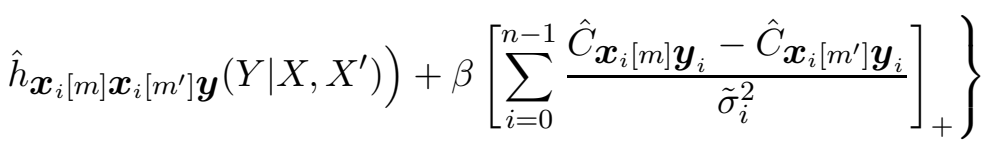

$$
\begin{aligned}
& \doteq \exp \left\{-\ell \inf _{\left\{\sigma_{\boldsymbol{y}_{i}}^{2}, \hat{C} \boldsymbol{x}_{i}[m] \boldsymbol{y}_{i}, \hat{C} \boldsymbol{x}_{i}\left[m^{\prime}\right] \boldsymbol{y}_{i}\right\}_{i}} \sum_{i=0}^{n-1}\left(\frac{1}{2} \ln \left(2 \pi \sigma_{i}^{2}\right)+\frac{\hat{\sigma}_{\boldsymbol{y}_{i}}^{2}-2 \hat{C}_{\boldsymbol{x}_{i}[m] \boldsymbol{y}_{i}}+P_{i}}{2 \sigma_{i}^{2}}-\right.\right.
\end{aligned}
$$

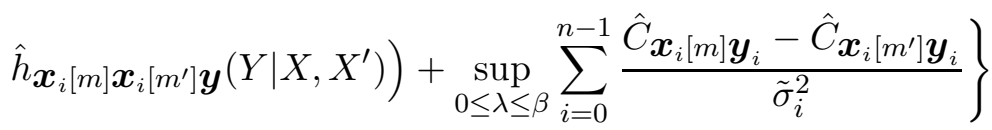

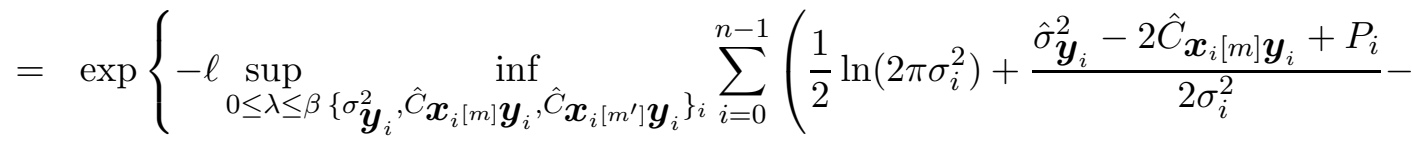

$$
\begin{aligned}
& \left.\left.\hat{h}_{\boldsymbol{x}_{i}[m] \boldsymbol{x}_{i}\left[m^{\prime}\right] \boldsymbol{y}}\left(Y \mid X, X^{\prime}\right)\right)+\sum_{i=0}^{n-1} \frac{\hat{C}_{\boldsymbol{x}_{i}[m]} \boldsymbol{y}_{i}-\hat{C}_{\boldsymbol{x}_{i}\left[m^{\prime}\right] \boldsymbol{y}_{i}}}{\tilde{\sigma}_{i}^{2}}\right\} \\
& =\exp \left\{-\ell \sup _{0 \leq \lambda \leq \beta} \sum_{i=0}^{n-1} \inf _{\left\{\sigma_{\boldsymbol{y}_{i}}^{2}, \hat{C} \boldsymbol{x}_{i}[m] \boldsymbol{y}_{i}, \hat{C} \boldsymbol{x}_{i\left[m^{\prime}\right]} \boldsymbol{y}_{i}\right\}_{i}}\left(\frac{1}{2} \ln \left(2 \pi \sigma_{i}^{2}\right)+\frac{\hat{\sigma}_{\boldsymbol{y}_{i}}^{2}-2 \hat{C}_{\boldsymbol{x}_{i}[m] \boldsymbol{y}_{i}+P_{i}}}{2 \sigma_{i}^{2}}-\right.\right. \\
& \left.\left.\hat{h}_{\boldsymbol{x}_{i}[m] \boldsymbol{x}_{i}\left[m^{\prime}\right] \boldsymbol{y}}\left(Y \mid X, X^{\prime}\right)+\sum_{i=0}^{n-1} \frac{\hat{C}_{\boldsymbol{x}_{i}[m]} \boldsymbol{y}_{i}-\hat{C}_{\boldsymbol{x}_{i}\left[m^{\prime}\right] \boldsymbol{y}_{i}}}{\tilde{\sigma}_{i}^{2}}\right)\right\} \\
& =\exp \left\{-\ell \sup _{0 \leq \lambda \leq \beta} \sum_{i=0}^{n-1} \lambda \mu_{i}\left(1-\lambda \mu_{i}\right) \operatorname{snr}_{i}\left(1-\rho_{i}\left[m, m^{\prime}\right]\right)\right\} \text {, }
\end{aligned}
$$

where $\rho_{i}\left[m, m^{\prime}\right]$ is the empirical correlation coefficient between $\boldsymbol{x}_{i}[m]$ and $\boldsymbol{x}_{i}\left[m^{\prime}\right]$, and where the last

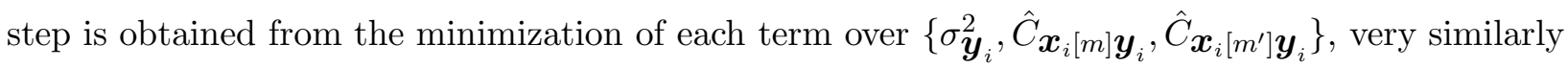


as is done in eq. (27). The proof is completed similarly as in [12] by showing that the typical number of codeword pairs $\left\{\boldsymbol{x}[m], \boldsymbol{x}\left[m^{\prime}\right]\right\}$ for which the segmental empirical correlation coefficients are around $\left(\rho_{0}, \rho_{1}, \ldots, \rho_{n-1}\right)$ (within a fine grid) is of the exponential order of

$$
M\left(\rho_{0}, \rho_{1}, \ldots, \rho_{n-1}\right) \doteq \exp \left\{N\left[2 R-\frac{1}{2 n} \sum_{i=0}^{n-1} \ln \frac{1}{1-\rho_{i}^{2}}\right]\right\}
$$

as long as $\frac{1}{2 n} \sum_{i=0}^{n-1} \ln \frac{1}{1-\rho_{i}^{2}}<2 R$, and $M\left(\rho_{0}, \rho_{1}, \ldots, \rho_{n-1}\right)=0$ otherwise. Thus, for the typical code,

$$
\begin{aligned}
P_{\mathrm{e}}\left(\mathcal{C}_{n}\right) \leq & \frac{1}{M} \sum_{m=0}^{M-1} \sum_{m^{\prime} \neq m} \exp \left\{-\ell \sup _{0 \leq \lambda \leq \beta} \sum_{i=0}^{n-1} \lambda \mu_{i}\left(1-\lambda \mu_{i}\right) \operatorname{snr}_{i}\left(1-\rho_{i}\left[m, m^{\prime}\right]\right)\right\} \\
\doteq & e^{-N R} \sum_{\rho_{0}, \rho_{1}, \ldots, \rho_{n-1}} M\left(\rho_{0}, \rho_{1}, \ldots, \rho_{n-1}\right) \times \\
& \exp \left\{-\ell \sup _{0 \leq \lambda \leq \beta} \sum_{i=0}^{n-1} \lambda \mu_{i}\left(1-\lambda \mu_{i}\right) \operatorname{snr}_{i}\left(1-\rho_{i}\right)\right\} \\
\doteq & \exp \left\{-N \inf _{\rho_{0}, \rho_{1}, \ldots, \rho_{n-1}} \sup _{0 \leq \lambda \leq \beta} \frac{1}{n} \sum_{i=0}^{n-1}\left[\lambda \mu_{i}\left(1-\lambda \mu_{i}\right) \operatorname{snr}_{i}\left(1-\rho_{i}\right)+\frac{1}{2} \ln \frac{1}{1-\rho_{i}^{2}}\right]-R\right\} \\
\doteq & \exp \left\{-N \sup _{0 \leq \lambda \leq \beta} \inf _{\rho_{0}, \rho_{1}, \ldots, \rho_{n-1}} \frac{1}{n} \sum_{i=0}^{n-1}\left[\lambda \mu_{i}\left(1-\lambda \mu_{i}\right) \operatorname{snr}_{i}\left(1-\rho_{i}\right)+\frac{1}{2} \ln \frac{1}{1-\rho_{i}^{2}}\right]-R\right\},
\end{aligned}
$$

where the infimum is subject to the constraint $\frac{1}{2 n} \sum_{i=0}^{n-1} \ln \frac{1}{1-\rho_{i}^{2}}<2 R$. This constrained minimization at the exponent can be presented as

$$
\begin{aligned}
& \inf _{\rho_{0}, \rho_{1}, \ldots, \rho_{n-1}} \sup _{\vartheta \geq 0} \frac{1}{n} \sum_{i=0}^{n-1}\left[\lambda \mu_{i}\left(1-\lambda \mu_{i}\right) \operatorname{snr}_{i}\left(1-\rho_{i}\right)+\frac{1}{2} \ln \frac{1}{1-\rho_{i}^{2}}+\vartheta\left(\frac{1}{2} \ln \frac{1}{1-\rho_{i}^{2}}-2 R\right)\right]-R \\
= & \sup _{\vartheta \geq 0} \frac{1}{n} \sum_{i=0}^{n-1} \inf _{\rho_{i}}\left[\lambda \mu_{i}\left(1-\lambda \mu_{i}\right) \operatorname{snr}_{i}\left(1-\rho_{i}\right)+\frac{1+\vartheta}{2} \ln \frac{1}{1-\rho_{i}^{2}}\right]-(2 \vartheta+1) R \\
= & \sup _{\theta \geq 1} \frac{1}{n} \sum_{i=0}^{n-1} \inf _{\rho_{i}}\left[\lambda \mu_{i}\left(1-\lambda \mu_{i}\right) \operatorname{snr}_{i}\left(1-\rho_{i}\right)+\frac{\theta}{2} \ln \frac{1}{1-\rho_{i}^{2}}\right]-(2 \theta-1) R \\
= & \sup _{\theta \geq 1} \frac{1}{n} \sum_{i=0}^{n-1} A\left(\operatorname{snr}_{i}, \mu_{i}, \lambda, \theta\right)-(2 \theta-1) R,
\end{aligned}
$$

which is the relevant expression for Theorem 2 .

\section{Appendix A: Proof of Eq. (82)}

For a given $\epsilon>0$, consider the set of vectors,

$$
H_{n}^{\epsilon}(B)=H_{n}(B) \bigcap\left\{\boldsymbol{y}=\left(i_{1}, i_{2}, \ldots, i_{n}\right) \cdot 2 \epsilon:\left(i_{1}, i_{2}, \ldots, i_{n}\right) \in \mathcal{Z}^{n}\right\}
$$


namely, the grid of all vectors within $H_{n}(B)$ whose components are integer multiples of $2 \epsilon$. Obviously,

$$
\left|H_{n}^{\epsilon}(B)\right| \leq \frac{\operatorname{Vol}\left\{H_{n}(B)\right\}}{(2 \epsilon)^{n}} \leq \frac{(2 \pi e B)^{n / 2}}{(2 \epsilon)^{n}}=\left(\frac{\sqrt{2 \pi e B}}{2 \epsilon}\right)^{n},
$$

in other words, the number of points within $H_{n}^{\epsilon}(B)$ is exponential in $n$. If we prove that

$$
\operatorname{Pr}\left\{\exists m \in\{0,1, \ldots, M-1\}, \boldsymbol{y} \in \mathcal{H}_{n}^{\epsilon}(B): Z_{m}(\boldsymbol{y})<\exp \left\{n \alpha\left(R-\epsilon, \hat{\sigma}_{\boldsymbol{y}}^{2}\right)\right\} \doteq e^{-n \infty}\right.
$$

then the result will follow by continuity considerations as follows: if $\boldsymbol{y}$ and $\boldsymbol{y}^{\prime}$ differ by no more than $\epsilon$ component-wise in absolute value, then for $\epsilon \ll 1 / \sqrt{B}$,

$$
\begin{aligned}
\hat{\sigma} \boldsymbol{y} & =\sqrt{\frac{1}{n}\|\boldsymbol{y}\|^{2}} \\
& \geq \sqrt{\frac{1}{n}\left(\left\|\boldsymbol{y}^{\prime}\right\|^{2}-2 \sqrt{B n} \sqrt{n \epsilon^{2}}+n \epsilon^{2}\right)} \\
& \geq \sqrt{\hat{\sigma}_{\boldsymbol{y}^{\prime}}^{2}-2 \sqrt{B} \epsilon} \\
& \geq \hat{\sigma} \boldsymbol{y}^{\prime}-\frac{2 \sqrt{B} \epsilon}{2 \hat{\sigma} \boldsymbol{y}^{\prime}} \\
& \geq \hat{\sigma} \boldsymbol{y}^{\prime}-\frac{2 \sqrt{B} \epsilon}{2 / \sqrt{B}} \\
& =\hat{\sigma} \boldsymbol{y}^{\prime}-B \epsilon .
\end{aligned}
$$

Thus,

$$
\begin{aligned}
\alpha\left(R, \hat{\sigma}_{\boldsymbol{y}}^{2}\right) & =\sup _{|\rho| \leq \sqrt{1-e^{-2 R}}}\left[\frac{\beta \sqrt{P} \hat{\sigma} \boldsymbol{y} \rho}{\sigma^{2}}+\frac{1}{2} \ln \left(1-\rho^{2}\right)\right]+R \\
& \geq \sup _{|\rho| \leq \sqrt{1-e^{-2 R}}}\left[\frac{\beta \sqrt{P}\left(\hat{\sigma}_{\boldsymbol{y}^{\prime}}-B \epsilon\right) \rho}{\sigma^{2}}+\frac{1}{2} \ln \left(1-\rho^{2}\right)\right]+R \\
& \geq \alpha\left(R, \hat{\sigma}_{\boldsymbol{y}^{\prime}}^{2}\right)-\frac{\beta \sqrt{P} B \epsilon}{\sigma^{2}} .
\end{aligned}
$$

On the other hand,

$$
\begin{aligned}
Z_{m}(\boldsymbol{y}) & =\sum_{m^{\prime} \neq m} \exp \left\{\frac{\beta \boldsymbol{x}^{T}\left[m^{\prime}\right] \boldsymbol{y}}{\sigma^{2}}\right\} \\
& \leq \sum_{m^{\prime} \neq m} \exp \left\{\frac{\beta\left(\boldsymbol{x}^{T}\left[m^{\prime}\right] \boldsymbol{y}^{\prime}+\sqrt{n P} \sqrt{n \epsilon^{2}}\right)}{\sigma^{2}}\right\} \\
& =\exp \left\{\frac{n \beta \sqrt{P} \epsilon}{\sigma^{2}}\right\} \cdot Z_{m}\left(\boldsymbol{y}^{\prime}\right) .
\end{aligned}
$$


It follows that if $Z_{m}(\boldsymbol{y}) \geq \exp \left\{n \alpha\left(R-\epsilon, \hat{\sigma}_{\boldsymbol{y}}^{2}\right)\right.$ for all $(m, \boldsymbol{y}) \in\{0,1, \ldots, M-1\} \times \mathcal{H}_{n}^{\epsilon}(B)$, then $Z_{m}(\boldsymbol{y}) \geq \exp \left\{n\left[\alpha\left(R-\epsilon, \hat{\sigma}_{\boldsymbol{y}}^{2}\right)-\delta(\epsilon)\right]\right\}$ for all $(m, \boldsymbol{y}) \in\{0,1, \ldots, M-1\} \times \mathcal{H}_{n}(B)$, where $\delta(\epsilon)$ is as defined above.

Since the number of pairs $(m, \boldsymbol{y}) \in\{0,1, \ldots, M-1\} \times \mathcal{H}_{n}^{\epsilon}(B)$ is upper bounded by $e^{n R}$. $(\sqrt{2 \pi e B} / \epsilon)^{n}$, which is exponential in $n$, it is enough to prove that

$$
\operatorname{Pr}\left\{Z_{m}(\boldsymbol{y})<\exp \left\{n \alpha\left(R-\epsilon, \hat{\sigma}_{\boldsymbol{y}}^{2}\right)\right\} \doteq e^{-n \infty}\right.
$$

for every given $(m, \boldsymbol{y}) \in\{0,1, \ldots, M-1\} \times \mathcal{H}_{n}^{\epsilon}(B)$, as the union bound over all these pairs will not affect the super-exponential decay of the probability under discussion. The proof of this fact is the same as the proof of the analogous result in [13, Appendix B], except that here $Z_{m}(\boldsymbol{y})$ is approximated as $\sum_{i} M\left(\rho_{i}\right) \exp \left\{\beta n \sqrt{P} \hat{\sigma} \boldsymbol{y} \rho_{i} / \sigma^{2}\right\}$, where $\left\{\rho_{i}\right\}$ form a fine quantization grid with spacing $\epsilon$ within the interval $(-1,1)$ and $M\left(\rho_{i}\right)$ is the number of codewords other than $\boldsymbol{x}[m]$ whose empirical correlation coefficient with $\boldsymbol{y}$ is between $\rho_{i}-\epsilon / 2$ and $\rho_{i}+\epsilon / 2$. The proof in $[13$, Appendix B] applies here verbatim except that $Q, g(Q)$ and $I(Q)$ of [13] are replaced by $\rho_{i}, \beta \sqrt{P} \hat{\sigma} \boldsymbol{y} \rho_{i}$ and $\frac{1}{2} \ln \frac{1}{1-\rho_{i}^{2}}$, respectively.

\section{Appendix B: Calculation of $R_{0}$}

In ordinary random coding under the same regime, we have the following. The pairwise error event is:

$$
\sum_{i=0}^{n-1} \frac{\tilde{\boldsymbol{x}}_{i}^{T} \boldsymbol{y}_{i}}{\tilde{\sigma}_{i}^{2}} \geq \sum_{i=0}^{n-1} \frac{\boldsymbol{x}_{i}^{T} \boldsymbol{y}_{i}}{\tilde{\sigma}_{i}^{2}}
$$

where $\boldsymbol{x}_{i}$ represents the $i$-th segment of the transmitted codeword and $\tilde{\boldsymbol{x}}_{i}$ stands for that of a competing one. On substituting $\boldsymbol{y}_{i}=\boldsymbol{x}_{i}+\boldsymbol{z}_{i}$, this becomes

$$
\sum_{i=0}^{n-1} \frac{\tilde{\boldsymbol{x}}_{i}^{T}\left(\boldsymbol{x}_{i}+\boldsymbol{z}_{i}\right)}{\tilde{\sigma}_{i}^{2}} \geq \sum_{i=0}^{n-1} \frac{\boldsymbol{x}_{i}^{T}\left(\boldsymbol{x}_{i}+\boldsymbol{z}_{i}\right)}{\tilde{\sigma}_{i}^{2}}
$$

or

$$
\sum_{i=0}^{n-1} \frac{\left(\tilde{\boldsymbol{x}}_{i}-\boldsymbol{x}_{i}\right)^{T} \boldsymbol{z}_{i}}{\tilde{\sigma}_{i}^{2}} \geq \ell \sum_{i=0}^{n-1} \frac{P_{i}\left(1-\rho_{i}\right)}{\tilde{\sigma}_{i}^{2}},
$$

where $\rho_{i}$ is the empirical correlation coefficient between $\boldsymbol{x}_{i}$ and $\tilde{\boldsymbol{x}}_{i}$. Now,

$$
\sum_{i=0}^{n-1} \frac{\left(\tilde{\boldsymbol{x}}_{i}-\boldsymbol{x}_{i}\right)^{T} \boldsymbol{z}_{i}}{\tilde{\sigma}_{i}^{2}} \sim \mathcal{N}\left(0,2 \ell \sum_{i=0}^{n-1} \frac{P_{i} \sigma_{i}^{2}\left(1-\rho_{i}\right)}{\tilde{\sigma}_{i}^{4}}\right),
$$


thus, the probability of the above event is

$$
\begin{aligned}
\operatorname{Pr}\left\{\sum_{i=0}^{n-1} \frac{\left(\tilde{\boldsymbol{x}}_{i}-\boldsymbol{x}_{i}\right)^{T} \boldsymbol{z}_{i}}{\tilde{\sigma}_{i}^{2}} \geq \ell \sum_{i=0}^{n-1} \frac{P_{i}\left(1-\rho_{i}\right)}{\tilde{\sigma}_{i}^{2}}\right\} & =Q\left(\frac{\ell \sum_{i=0}^{n-1} P_{i}\left(1-\rho_{i}\right) / \tilde{\sigma}_{i}^{2}}{\sqrt{2 \ell \sum_{i=0}^{n-1} P_{i} \sigma_{i}^{2}\left(1-\rho_{i}\right) / \tilde{\sigma}_{i}^{4}}}\right) \\
& \doteq \exp \left\{-\frac{\ell\left[\sum_{i=0}^{n-1} P_{i}\left(1-\rho_{i}\right) / \tilde{\sigma}_{i}^{2}\right]^{2}}{4 \sum_{i=0}^{n-1} P_{i} \sigma_{i}^{2}\left(1-\rho_{i}\right) / \tilde{\sigma}_{i}^{4}}\right\},
\end{aligned}
$$

where $Q(\cdot)$ is the error function,

$$
Q(x)=\frac{1}{\sqrt{2 \pi}} \int_{x}^{\infty} e^{-u^{2} / 2} \mathrm{~d} u .
$$

The average pairwise probability of error is therefore given by

$$
\begin{aligned}
& \overline{\operatorname{Pr}}\left\{\sum_{i=0}^{n-1} \frac{\left(\tilde{\boldsymbol{x}}_{i}-\boldsymbol{x}_{i}\right)^{T} \boldsymbol{z}_{i}}{\tilde{\sigma}_{i}^{2}} \geq \ell \sum_{i=0}^{n-1} \frac{P_{i}\left(1-\rho_{i}\right)}{\tilde{\sigma}_{i}^{2}}\right\} \\
\doteq & \max _{\rho_{1}, \ldots, \rho_{n}} \exp \left\{\ell \sum_{i=0}^{n-1} \frac{1}{2} \ln \left(1-\rho_{i}^{2}\right)-\frac{\ell\left[\sum_{i=0}^{n-1} P_{i}\left(1-\rho_{i}\right) / \tilde{\sigma}_{i}^{2}\right]^{2}}{4 \sum_{i=0}^{n-1} P_{i} \sigma_{i}^{2}\left(1-\rho_{i}\right) / \tilde{\sigma}_{i}^{4}}\right\},
\end{aligned}
$$

where the term $\ell \sum_{i=0}^{n-1} \frac{1}{2} \ln \left(1-\rho_{i}^{2}\right)$ accounts for the probabilistic weight of $\left(\rho_{0}, \ldots, \rho_{n-1}\right)$. Therefore, the exponent is

$$
\begin{aligned}
& \min _{\rho_{0}, \ldots, \rho_{n-1}}\left\{\frac{1}{n} \sum_{i=0}^{n-1} \frac{1}{2} \ln \frac{1}{1-\rho_{i}^{2}}+\frac{\left[\sum_{i=0}^{n-1} P_{i}\left(1-\rho_{i}\right) / \tilde{\sigma}_{i}^{2}\right]^{2}}{4 n \sum_{i=0}^{n-1} P_{i} \sigma_{i}^{2}\left(1-\rho_{i}\right) / \tilde{\sigma}_{i}^{4}}\right\} \\
= & \min _{\rho_{0}, \ldots, \rho_{n-1}}\left\{-\frac{1}{n} \sum_{i=0}^{n-1} \frac{1}{2} \ln \left(1-\rho_{i}^{2}\right)+\sup _{\lambda \geq 0}\left[\lambda \cdot \frac{1}{n} \sum_{i=0}^{n-1} \frac{P_{i}\left(1-\rho_{i}\right)}{\tilde{\sigma}_{i}^{2}}-\lambda^{2} \cdot \frac{1}{n} \sum_{i=0}^{n-1} \frac{P_{i} \sigma_{i}^{2}\left(1-\rho_{i}\right)}{\tilde{\sigma}_{i}^{4}}\right]\right\} \\
= & \sup _{\lambda \geq 0} \frac{1}{n} \sum_{i=0}^{n-1} \min _{\rho_{i}}\left\{\frac{1}{2} \ln \frac{1}{1-\rho_{i}^{2}}+\lambda \cdot \frac{P_{i}\left(1-\rho_{i}\right)}{\tilde{\sigma}_{i}^{2}}-\lambda^{2} \cdot \frac{P_{i} \sigma_{i}^{2}\left(1-\rho_{i}\right)}{\tilde{\sigma}_{i}^{4}}\right\} \\
= & \sup _{\lambda \geq 0} \frac{1}{n} \sum_{i=0}^{n-1} \min _{\rho_{i}}\left\{\frac{1}{2} \ln \frac{1}{1-\rho_{i}^{2}}+\frac{P_{i}}{\tilde{\sigma}_{i}^{2}}\left(\lambda-\frac{\lambda^{2} \sigma_{i}^{2}}{\tilde{\sigma}_{i}^{2}}\right)\left(1-\rho_{i}\right)\right\} \\
= & \sup _{\lambda \geq 0} \frac{1}{n} \sum_{i=0}^{n-1} A\left(\operatorname{snr}_{i}, \mu_{i}, \lambda, 1\right),
\end{aligned}
$$

and in the limit of large $n$,

$$
R_{0}=\sup _{\lambda \geq 0} \frac{1}{2 \pi} \int_{0}^{2 \pi} A[\operatorname{snr}(\omega), \mu(\omega), \lambda, 1] \mathrm{d} \omega=B(1),
$$

as opposed to the zero-rate TRC exponent, which corresponds to

$$
\sup _{\lambda \geq 0} \frac{1}{2 \pi} \int_{0}^{2 \pi} A[\operatorname{snr}(\omega), \mu(\omega), \lambda, \infty] \mathrm{d} \omega .
$$




\section{Appendix C: Tightness at Low Rates}

As before, we begin from parallel channels, and then take the limit $n \rightarrow \infty$ in order to pass to the continuous frequency domain. For deterministic decoding we also take the limit $\beta \rightarrow \infty$. Let us denote

$$
G(\boldsymbol{x}, \boldsymbol{y})=\frac{1}{n} \sum_{i=0}^{n-1} \frac{\boldsymbol{x}_{i}^{T} \boldsymbol{y}_{i}}{\tilde{\sigma}_{i}^{2}}
$$

and

$$
\alpha(R, \boldsymbol{y})=\sup \sum_{i=0}^{n-1} \frac{\sqrt{P_{i}} \hat{\sigma}_{\boldsymbol{y}_{i}} \rho_{i}}{\tilde{\sigma}_{i}^{2}},
$$

where the supermum is over all $\left(\rho_{0}, \rho_{1}, \ldots, \rho_{n-1}\right)$ such that $\sum_{i=0}^{n-1} \frac{1}{2} \ln \frac{1}{1-\rho_{i}^{2}} \leq n R$. As in [12], the TRC exponent for deterministic mismatched decoding is obtained by analyzing the probability of the event $G\left(\boldsymbol{x}_{m^{\prime}}, \boldsymbol{y}\right) \geq \max \left\{G\left(\boldsymbol{x}_{m}, \boldsymbol{y}\right), \alpha(R, \boldsymbol{y})\right\}$, for a given code, taking the logarithm, and finally, averaging over the code ensemble. As mentioned earlier, here we removed the term $\alpha(\boldsymbol{y}, R)$ and upper bounded by this probability by the probability of the event $G\left(\boldsymbol{x}_{m^{\prime}}, \boldsymbol{y}\right) \geq G\left(\boldsymbol{x}_{m}, \boldsymbol{y}\right)$, which is the union of pairwise error events. We would like to show now that there is a range of low rates $\left[0, R_{\mathrm{t}}\right]$, where this bound is exponentially tight. Consider the chain of inequalities

$$
\begin{aligned}
& \operatorname{Pr}\left\{G\left(\boldsymbol{x}\left[m^{\prime}\right], \boldsymbol{y}\right) \geq G(\boldsymbol{x}[m], \boldsymbol{y})\right\} \\
= & \operatorname{Pr}\left\{G\left(\boldsymbol{x}\left[m^{\prime}\right], \boldsymbol{y}\right) \geq G(\boldsymbol{x}[m], \boldsymbol{y}) \geq \alpha(R, \boldsymbol{y})\right\}+\operatorname{Pr}\left\{G\left(\boldsymbol{x}\left[m^{\prime}\right], \boldsymbol{y}\right) \geq \alpha(R, \boldsymbol{y}) \geq G(\boldsymbol{x}[m], \boldsymbol{y})\right\}+ \\
& \operatorname{Pr}\left\{\alpha(R, \boldsymbol{y}) \geq G\left(\boldsymbol{x}\left[m^{\prime}\right], \boldsymbol{y}\right) \geq G(\boldsymbol{x}[m], \boldsymbol{y})\right\} \\
= & \operatorname{Pr}\left\{G\left(\boldsymbol{x}\left[m^{\prime}\right], \boldsymbol{y}\right) \geq \max \{\alpha(R, \boldsymbol{y}), G(\boldsymbol{x}[m], \boldsymbol{y})\}+\operatorname{Pr}\left\{\alpha(R, \boldsymbol{y}) \geq G\left(\boldsymbol{x}\left[m^{\prime}\right], \boldsymbol{y}\right) \geq G(\boldsymbol{x}[m], \boldsymbol{y})\right\}\right. \\
\leq & \operatorname{Pr}\left\{G\left(\boldsymbol{x}\left[m^{\prime}\right], \boldsymbol{y}\right) \geq \max \{\alpha(R, \boldsymbol{y}), G(\boldsymbol{x}[m], \boldsymbol{y})\}+\operatorname{Pr}\{\alpha(R, \boldsymbol{y}) \geq G(\boldsymbol{x}[m], \boldsymbol{y})\},\right.
\end{aligned}
$$

where the left-most side is the quantity we analyze in the proof of Theorem 2, and in the rightmost side, the first term is the desired quantity, whereas the second term is the supplementary term that we would now like to focus on. In particular, if we show that the second term decays at an exponential rate faster than that of the first term (which is $E_{\mathrm{trc}}^{-}(R)$ ), then our union-bound analysis in Theorem 2 is tight. Now,

$$
\operatorname{Pr}\{\alpha(R, \boldsymbol{y}) \geq G(\boldsymbol{x}[m], \boldsymbol{y})\}=\operatorname{Pr}\left\{\max _{\left\{-\sum_{i=0}^{n-1} \ln \left(1-\hat{\rho}_{i}^{2}\right) \leq 2 n R\right\}} \sum_{i=0}^{n-1} \frac{\sqrt{P_{i}} \hat{\sigma}_{\boldsymbol{y}_{i}} \hat{\rho}_{i}}{\tilde{\sigma}_{i}^{2}} \geq \frac{1}{\ell} \sum_{i=0}^{n-1} \frac{\boldsymbol{x}_{i}^{T}[m] \boldsymbol{y}_{i}}{\tilde{\sigma}_{i}^{2}}\right\} .
$$


But

$$
\begin{aligned}
& \max _{\left\{-\sum_{i=0}^{n-1} \max \left(1-\hat{\rho}_{i}^{2}\right) \leq 2 n R\right\}} \sum_{i} \frac{\sqrt{P_{i}} \hat{\sigma}_{i} \hat{\rho}_{i}}{\tilde{\sigma}_{i}^{2}} \\
= & \max _{\hat{\rho}_{0}, \ldots, \hat{\rho}_{n-1}} \inf _{\lambda \geq 0} \sum_{i=0}^{n-1}\left\{\frac{\sqrt{P_{i}} \hat{\sigma} \boldsymbol{y}_{i} \hat{\rho}_{i}}{\tilde{\sigma}_{i}^{2}}+\lambda\left[R+\frac{1}{2} \ln \left(1-\hat{\rho}_{i}^{2}\right)\right]\right\} \\
= & \inf _{\lambda \geq 0} \sum_{0=1}^{n-1} \max _{\rho_{i}}\left\{\frac{\sqrt{P_{i}} \hat{\sigma}_{\boldsymbol{y}_{i}} \hat{\rho}_{i}}{\tilde{\sigma}_{i}^{2}}+\lambda\left[R+\frac{1}{2} \ln \left(1-\hat{\rho}_{i}^{2}\right)\right]\right\} \\
= & \inf _{\lambda \geq 0}\left\{\sum_{0=1}^{n-1} W\left(\frac{\sqrt{P_{i}} \hat{\sigma} \boldsymbol{y}_{i}}{\tilde{\sigma}_{i}^{2}}, \lambda\right)+\lambda R\right\},
\end{aligned}
$$

where we define

$$
\begin{aligned}
W(\alpha, \lambda) & =\max _{\rho}\left\{\alpha \rho+\frac{\lambda}{2} \ln \left(1-\rho^{2}\right)\right\} \\
& =\lambda \cdot w\left(\frac{\alpha}{\lambda}, \infty\right) \\
& =\frac{2 \alpha^{2}}{\lambda+\sqrt{\lambda^{2}+4 \alpha^{2}}}+\frac{\lambda}{2} \ln \left[\frac{2 \lambda}{\lambda+\sqrt{\lambda^{2}+4 \alpha^{2}}}\right] .
\end{aligned}
$$

Now,

$$
\begin{aligned}
\operatorname{Pr}\{\alpha(R, \boldsymbol{y}) \geq G(\boldsymbol{x}[m], \boldsymbol{y})\} & =\operatorname{Pr}\left\{\frac{1}{\ell} \sum_{i=0}^{n-1} \frac{\boldsymbol{x}_{i}^{T}[m] \boldsymbol{y}_{i}}{\tilde{\sigma}_{i}^{2}} \leq \inf _{\lambda \geq 0}\left[\sum_{i=1}^{k} W\left(\frac{\sqrt{P_{i}} \hat{\sigma} \boldsymbol{y}_{i}}{\tilde{\sigma}_{i}^{2}}, \lambda\right)+\lambda R\right]\right\} \\
& \leq \inf _{\lambda \geq 0} \operatorname{Pr}\left\{\frac{1}{\ell} \sum_{i=0}^{n-1} \frac{\boldsymbol{x}_{i}^{T}[m] \boldsymbol{y}_{i}}{\tilde{\sigma}_{i}^{2}} \leq \sum_{i=0}^{n-1}\left[W\left(\frac{\sqrt{P_{i}} \hat{\sigma}_{i}}{\tilde{\sigma}_{i}^{2}}, \lambda\right)+\lambda R\right]\right\}
\end{aligned}
$$

For a given $\lambda \geq 0$, the exponent of the last probability is given by

$$
\min _{\left\{\rho_{i}, \hat{\sigma}_{\boldsymbol{y}_{i}}^{2}\right\}} \frac{1}{n} \sum_{i=0}^{n-1}\left\{\frac{1}{2} \ln \left(2 \pi \sigma_{i}^{2}\right)+\frac{\hat{\sigma}_{\boldsymbol{y}_{i}}^{2}-2 \sqrt{P_{i}} \hat{\sigma} \boldsymbol{y}_{i} \rho_{i}+P_{i}}{2 \sigma_{i}^{2}}-\frac{1}{2} \ln \left[2 \pi e \hat{\sigma}_{\boldsymbol{y}_{i}}^{2}\left(1-\rho_{i}^{2}\right)\right]\right\},
$$

where the minimum is taken over all $\left\{\left(\rho_{i}, \hat{\sigma}_{\boldsymbol{y}_{i}}^{2}\right), 0=1,2, \ldots, n-1\right\}$ such that

$$
\sum_{i=0}^{n-1} \frac{\sqrt{P_{i}} \hat{\sigma}_{\boldsymbol{y}_{i}} \rho_{i}}{\tilde{\sigma}_{i}^{2}} \leq \sum_{i=0}^{n-1}\left[W\left(\frac{\sqrt{P_{i}} \hat{\sigma} \boldsymbol{y}_{i}}{\tilde{\sigma}_{i}^{2}}, \lambda\right)+\lambda R\right]
$$

or, equivalently, the exponent is given by

$$
\begin{aligned}
& \min _{\left\{\rho_{i}, \hat{\sigma}_{\boldsymbol{y}_{i}}^{2}\right\}} \sup _{\zeta \geq 0} \frac{1}{n} \sum_{0=1}^{n-1}\left\{\frac{1}{2} \ln \left(2 \pi \sigma_{i}^{2}\right)+\frac{\hat{\sigma}_{\boldsymbol{y}_{i}}^{2}-2 \sqrt{P_{i}} \hat{\sigma}_{\boldsymbol{y}_{i}} \rho_{i}+P_{i}}{2 \sigma_{i}^{2}}-\frac{1}{2} \ln \left[2 \pi e \hat{\sigma}_{\boldsymbol{y}_{i}}^{2}\left(1-\rho_{i}^{2}\right)\right]+\right. \\
& \left.\frac{\zeta}{\lambda}\left[\frac{\sqrt{P_{i}} \hat{\sigma} \boldsymbol{y}_{i} \rho_{i}}{\tilde{\sigma}_{i}^{2}}-W\left(\frac{\sqrt{P_{i}} \hat{\sigma} \boldsymbol{y}_{i}}{\tilde{\sigma}_{i}^{2}}, \lambda\right)-\lambda R\right]\right\}
\end{aligned}
$$




$$
\begin{aligned}
\geq & \sup _{\zeta \geq 0} \frac{1}{n} \sum_{i=0}^{n-1} \min _{\rho_{i}, \hat{\sigma}_{\boldsymbol{y}_{i}}}\left\{\frac{1}{2} \ln \left(2 \pi \sigma_{i}^{2}\right)+\frac{\hat{\sigma}_{\boldsymbol{y}_{i}}^{2}-2 \sqrt{P_{i}} \hat{\sigma}_{\boldsymbol{y}_{i}} \rho_{i}+P_{i}}{2 \sigma_{i}^{2}}-\frac{1}{2} \ln \left[2 \pi e \hat{\sigma}_{\boldsymbol{y}_{i}}^{2}\left(1-\rho_{i}^{2}\right)\right]+\right. \\
& \left.\frac{\zeta}{\lambda}\left[\frac{\sqrt{P_{i}} \hat{\sigma}_{\boldsymbol{y}_{i}} \rho_{i}}{\tilde{\sigma}_{i}^{2}}-W\left(\frac{\sqrt{P_{i}} \hat{\sigma} \boldsymbol{y}_{i}}{\tilde{\sigma}_{i}^{2}}, \lambda\right)-\lambda R\right]\right\} \\
\triangleq & \sup _{\zeta \geq 0} \frac{1}{n} \sum_{i=0}^{n-1} D\left(P_{i}, \sigma_{i}^{2}, \tilde{\sigma}_{i}^{2}, \lambda, \zeta\right)-\zeta R,
\end{aligned}
$$

where we have defined

$$
\begin{aligned}
D\left(P, \sigma^{2}, \tilde{\sigma}^{2}, \lambda, \zeta\right)= & \min _{\rho, \sigma_{Y}^{2}}\left\{\frac{1}{2} \ln \left(2 \pi \sigma^{2}\right)+\frac{\sigma_{Y}^{2}-2 \sqrt{P} \sigma_{Y} \rho+P}{2 \sigma^{2}}-\frac{1}{2} \ln \left[2 \pi e \sigma_{Y}^{2}\left(1-\rho^{2}\right)\right]+\right. \\
& \left.\frac{\zeta}{\lambda}\left[\frac{\sqrt{P} \sigma_{Y} \rho}{\tilde{\sigma}^{2}}-W\left(\frac{\sqrt{P} \sigma_{Y}}{\tilde{\sigma}^{2}}, \lambda\right)\right]\right\} .
\end{aligned}
$$

After the minimization over $\lambda \geq 0$, and after taking the limit of $n \rightarrow \infty$, we obtain

$$
\begin{aligned}
\varepsilon(R) & =\sup _{\zeta \geq 0}\left[\sup _{\lambda \geq 0} \frac{1}{2 \pi} \int_{0}^{2 \pi} D\left[S_{X}(\omega), S_{Z}(\omega), \tilde{S}_{Z}(\omega), \lambda, \zeta\right] \mathrm{d} \omega-\zeta R\right] \\
& \triangleq \sup _{\zeta \geq 0}[\Delta(\zeta)-\zeta R]
\end{aligned}
$$

and it is not difficult to check that $\varepsilon(0)=2 E_{\mathrm{trc}}^{-}(0)$, as for $R=0$, the optimum $\lambda$ tends to infinity, in which case, $W\left(\sqrt{P} \sigma_{Y} / \tilde{\sigma}^{2}, \lambda\right)$ vanishes. Thus $R_{\mathrm{t}}$, the maximum guaranteed rate of tightness of our TRC bound, is the supremum of all rates $R$ such that $\varepsilon(R)>E_{\mathrm{trc}}(R)$. To find an expression for $R_{*}$, we require that for every $\theta \geq 1$, there exists $\zeta>2 \theta-1$ such that

$$
\Delta(\zeta)-\zeta R \geq B(\theta)-(2 \theta-1) R
$$

or equivalently,

$$
R \leq \frac{\Delta(\zeta)-B(\theta)}{\zeta-2 \theta+1}
$$

Therefore,

$$
R_{\mathrm{t}}=\inf _{\theta \geq 1} \sup _{\zeta>2 \theta-1} \frac{\Delta(\zeta)-B(\theta)}{\zeta-2 \theta+1}
$$




\section{References}

[1] A. Barg and G. D. Forney, Jr., "Random codes: minimum distances and error exponents," IEEE Trans. Inform. Theory, vol. 48, no. 9, pp. 2568-2573, September 2002.

[2] G. Battail, "On random-like codes," Proc. 4th Canadian Workshop on Information Theory, pp. 76-94, Lac Delage, Quebec, Canada, May 1995.

[3] I. Csiszár and J. Körner, Information Theory: Coding Theorems for Discrete Memoryless Systems, Second Edition, Cambridge University Press, 2011.

[4] I. Csiszár and J. Körner, "Graph decomposition: a new key to coding theorems," IEEE Trans. Inform. Theory, vol. IT-27, no. 1, pp. 5-12, January 1981.

[5] P. M. Ebert, "Error bounds for parallel communication channels," M.I.T. Research Laboratory of Electronics, Technical Report no. 448, August, 1966.

[6] R. G. Gallager, Information Theory and Reliable Communication, John Wiley \& Sons, New York, 1968.

[7] R. M. Gray, "Toeplitz and circulant matrices: a review," Foundations and Trends in Communications and Information Theory, vol. 2, no. 3, pp. 155-239, 2006. http://dx.doi.org/10.1561/0100000006.

[8] U. Grenander and G. Szego, Toeplitz forms and their applications, University of California Press, Berkeley, 1958.

[9] Y. Kabashima, "How could the replica method improve accuracy of performance assessment of channel coding?" Proc. Int. Workshop on Statistical-Mechanical Informatics, Sept. 14-17, 2008, Sendai, Japan. arXiv:0808.0548v1 [cs.IT] 5 Aug 2008

[10] N. Merhav, "Universal decoding for memoryless Gaussian channels with a deterministic interference," IEEE Trans. Inform. Theory, vol. 39, no. 4, pp. 1261-1269, July 1993.

[11] N. Merhav, "Statistical physics and information theory," (invited paper) Foundations and Trends in Communications and Information Theory, vol. 6, nos. 1-2, pp. 1-212, 2009. 
[12] N. Merhav, "Error exponents of typical random codes," IEEE Trans. Inform. Theory, vol. 64, no. 9, pp. 6223-6235, September 2018.

[13] N. Merhav, "The generalized stochastic likelihood decoder: random coding and expurgated bounds," IEEE Trans. Inform. Theory, vol. 63, no. 8, pp. 5039-5051, August 2017.

[14] M. Mézard and A. Montanari, Information, Physics, and Computation, Oxford University Press, 2009.

[15] T. Mora and O. Rivoire, "Statistical mechanics of error exponents for error-correcting codes," Phys. Rev. E, vol. 74 056110, 2006.

[16] A. Nazari, Error exponent for discrete memoryless multiple-access channels, Ph.D. dissertation, Department of Electrical Engineering - Systems, the University of Michigan, 2011.

[17] A. Nazari, A. Anastasopoulos, and S. S. Pradhan, "Error exponent for multiple-access channels: lower bounds," IEEE Trans. Inform. Theory, vol. 60, no. 9, pp. 5095-5115, September 2014 .

[18] I. Sason and S. Shamai, "Performance analysis of linear codes under maximum-likelihood decoding: a tutorial," Foundations and Trends in Communications and Information Theory, vol. 3, no. 1/2, pp. 1-222, 2006.

[19] J. Scarlett, A. Martinéz and A. Guillén i Fábrigas, "The likelihood decoder: error exponents and mismatch," Proc. 2015 IEEE International Symposium on Information Theory (ISIT 2015), pp. 86-90, Hong Kong, June 2015.

[20] C. E. Shannon, "Probability of error for optimal codes in a Gaussian channel," the Bell System Technical Journal, vol. 38, no. 3, pp. 611-656, May 1959.

[21] C. E. Shannon, R. G. Gallager, and E. R. Berlekamp, "Lower bounds on the error probability for coding on discrete memoryless channels," Information and Control, vol. 10, pp. 65-103 (part I), pp. 522-552 (part II), 1967.

[22] N. S. Skantzos, J. van Mourik, Y. Kabashima, and D. Saad, "Average error exponent in Gallager low-density parity-check codes," J. Phys. A: Math. Gen., vol. 36, pp. 11131-11141, 2003. 
[23] D. Slepian, "Bounds on communication," the Bell System Technical Journal, vol. 42, pp. 681-707, 1963.

[24] A. J. Viterbi, "Error bounds for white Gaussian and other very noisy memoryless channels with generalized decision regions," IEEE Trans. Inform. Theory, vol. IT-15, no. 2, pp. 279287, March 1969.

[25] M. H. Yassaee, M. R. Aref and A. Gohari, "A technique for deriving one-shot achievability results in network information theory," [http://arxiv.org/abs/1303.0696], 2013. 